\title{
Akt Phosphorylates NQ01 and Triggers its Degradation, Abolishing Its Antioxidative Activities in Parkinson's Disease
}

\author{
@Shilin Luo, ${ }^{1,2}$ Seong Su Kang, ${ }^{1}$ Zhi-Hao Wang, ${ }^{1}$ Xia Liu, ${ }^{1}$ Julia X Day, ${ }^{3}$ Zhiping Wu, ${ }^{4}$ Junmin Peng, ${ }^{4}$ Daxiong Xiang, ${ }^{2}$ \\ (D)Wolfdieter Springer, ${ }^{5}$ and Keqiang $\mathrm{Ye}^{1}$ \\ ${ }^{1}$ Department of Pathology and Laboratory Medicine, Emory University School of Medicine, Atlanta, Georgia 30322, ${ }^{2}$ Department of Pharmacy, the Second \\ Xiangya Hospital, Central South University, Changsha, Hunan 410011, China, ${ }^{3}$ La Jolla Country Day School, La Jolla, California 92037, ${ }^{4}$ Departments of \\ Structural Biology \& Developmental Neurobiology, Center for Proteomics and Metabolomics, St. Jude Children's Research Hospital, Memphis, Tennessee \\ 38105, and ${ }^{5}$ Department of Neuroscience, Mayo Clinic College of Medicine and Science, Jacksonville, Florida 32224
}

The oxidative metabolism of dopamine and consequent oxidative stress are implicated in dopaminergic neuronal loss, mediating the pathogenesis of Parkinson's disease (PD). The inducible detoxifying antioxidative enzyme Quinone oxidoreductase (NQ01) (NAD(P)H: quinone oxidoreductase 1), neuroprotective to counteract reactive oxidative species, is most prominent in the active stage of the disease and virtually absent at the end stage of the disease. However, the molecular mechanism dictating NQO1 expression oscillation remains unclear. Here we show that Akt phosphorylates NQ01 at T128 residues and triggers its polyubiquitination and proteasomal degradation, abrogating its antioxidative effects in PD. Akt binds NQ01 in a phosphorylation-dependent manner. Interestingly, Akt, but not PINK1, provokes NQ01 phosphorylation and polyubiquitination with Parkin as an E3 ligase. Unphosphorylatable NQO1 mutant displays more robust neuroprotective activity than WT NQO1 in suppressing reactive oxidative species and against MPTP-induced dopaminergic cell death, rescuing the motor disorders in both $\alpha$-synuclein transgenic transgenic male and female mice elicited by the neurotoxin. Thus, our findings demonstrate that blockade of Akt-mediated NQO1 degradation may ameliorate PD pathogenesis.

Key words: Akt; antioxidative activity; NQ01; Parkinson's disease; phosphorylation; ubiquitination

Significance Statement

Dopaminergic neurodegeneration in Parkinson's disease (PD) is associated with the imbalance of oxidative metabolism of dopamine. Quinone oxidoreductase (NQO1), a potent antioxidant system, its expression levels are prominently increased in the early and intermediate stages of PD and disappeared in the end-stage PD. The molecular modification behavior of NQ01 after it is upregulated by oxidative stress in the early stage of PD, however, remains unclear. This study shows that Akt binds and phosphorylates NQ01 at T128 residue and promotes its ubiquitination and degradation, and Parkin acts as an E3 ligase in this process, which affects the antioxidant capacity of NQ01. This finding provides a novel molecular mechanism for NQ01 oscillation in PD pathogenesis.

\section{Introduction}

Parkinson's disease (PD) is the second most common neurodegenerative disease, characterized prominently at the cellular level

Received March 19, 2019; revised July 8, 2019; accepted July 15, 2019.

Author contributions: S.L. and K.Y. designed research; S.L., S.S.K., Z.-H.W., J.X.D., and Z.W. performed research; S.L., X.L., J.P., D.X., and K.Y. analyzed data; S.L., J.P., and W.S. edited the paper; K.Y. wrote the first draft of the paper.

This work was supported in part by National Institute of Aging, National Institutes of Health RF1AG051538 to K.Y. and R01AG047928 to J.P., the Rodent Behavioral Core (subsidized by the Emory University School of Medicine and one of the Emory Integrated (ore Facilities), the Viral Vector Core of the Emory Neuroscience National Institute of Neurological Disorders and Stroke Core Facilities P30NS055077, and Georgia Clinical \& Translational Science Alliance of the National Institutes of Health Award UL1TR002378.

The authors declare no competing financial interests. by progressive degeneration of neuromelanin-containing dopaminergic neurons in the substantia nigra (SN) pars compacta (Lang and Lozano, 1998). Mounting evidence suggests that oxidation of dopamine (DA) and consequent quinone modification and oxidative stress as major factors contributing to the vulnerability of DAergic cells (Hwang, 2013). Although DA is normally stored in vesicles, excess cytosolic DA is easily oxidized both spontaneously and enzymatically to produce DA quinone. Neu-

Correspondence should be addressed to Keqiang Ye at kye@emory.edu.

https://doi.org/10.1523/JNEUROSCI.0625-19.2019

Copyright $\odot 2019$ the authors 
romelanin formation is the outcome of a process generally known as DA autooxidation, a chain of oxidation reactions in which highly neurotoxic DA quinones are produced (Drukarch and van Muiswinkel, 2000). In both idiopathic and genetic cases of $\mathrm{PD}$, oxidative stress is thought to be the common underlying mechanism that leads to dopaminergic neuronal loss and motor disorders. As such, the SN of PD patients exhibit increased levels of oxidized lipids (Bosco et al., 2006), proteins and DNA (Nakabeppu et al., 2007), and decreased levels of reduced glutathione (GSH) (Spencer et al., 1998; Zeevalk et al., 2008).

The enzyme NAD $(\mathrm{P}) \mathrm{H}$ :quinone oxidoreductase (DT-diaphorase; NAD(P)H-(quinone acceptor) oxidoreductase; NQO1), a member of Phase II detoxification enzymes family, catalyzes the two-electron reduction of quinone to the redox-stable hydroquinone. Since DA and its metabolites have been implicated in the pathogenesis of $\mathrm{PD}$, NQO1 may exert a protective effect against such conditions. Accordingly, NQO1 protects against the damaging effects of cyclized quinones and oxidative stress induced during their redox cycling (Zafar et al., 2007). Induction of NQO1 by sulforaphane protected against neuro-cytotoxicity associated with DA quinone in vitro (Han et al., 2007) and against MPTP-elicited toxicity in vivo (Jazwa et al., 2011). Immunohistochemistry study reveals that NQO1 is distributed in dopaminergic neurons in both the SN and VTA and colocalized with TH (Schultzberg et al., 1988). Based on their clinical and neuropathological characteristics, NQO1 expression levels are prominently increased in the early and intermediate stages of PD and disappeared in the end-stage PD, when the loss of dopaminergic neurons is in an advanced state (van Muiswinkel et al., 2004). Thus, NQO1 is upregulated in the active phase of the disease when the degenerative process is accompanied by marked gliosis (Orr et al., 2002). Consequently, the induction of NQO1 constitutes part of a broad-spectrum neuroprotective response aimed to counteract DAQs and reactive oxidative species (ROS)-mediated toxicity (Duffy et al., 1998; Drukarch and van Muiswinkel, 2001; Graumann et al., 2002).

PI3-kinase/Akt signaling is generally considered neuroprotective, acting against stress conditions that occur during neurodegeneration. Surprisingly, Akt phosphorylates SRPK2 and enhances its catalytic activity, leading to the promotion of neuronal cell death in Alzheimer's disease (AD) (Jang et al., 2009). Moreover, Akt phosphorylates ataxin-1, leading to association with 14-3-3 that mediates the neurotoxicity of ataxin- 1 by stabilizing it. Therefore, Akt signaling and 14-3-3 cooperate to modulate the neurotoxicity of ataxin-1, providing insight into spinocerebellar ataxia Type 1 pathogenesis (Chen et al., 2003). Accumulating evidence shows defective Akt signaling in PD and in models of familial and sporadic PD (Greene et al., 2011). For instance, immunostaining of postmortem brains indicates that Akt phosphorylation at both S473 and T308 is significantly diminished in dopaminergic neurons in PD patients compared with non-PD patients (Malagelada et al., 2008; Timmons et al., 2009). Interestingly, we found that Akt directly binds NQO1 and phosphorylates it on both $\mathrm{S} 40$ and $\mathrm{T} 128$ residues, the latter being the major phosphorylation site. Noticeably, Akt, but not PINK1, phosphorylates NQO1 T128 and provokes its polyubiquitination and proteasomal degradation, which is mediated by the E3 ligase Parkin. Consequently, blockade of NQO1 phosphorylation in the S40A/T128A double mutant increases its stability and augments its antioxidative activity, resulting in protection against MPTPinduced dopaminergic neuronal loss and amelioration of motor dysfunctions. Hence, Akt displays a temporal role in mediating dopaminergic neuronal survival via phosphorylating the antioxidative machinery, including NQO1.

\section{Materials and Methods}

Antibodies and reagents. Antibodies to the following targets were used: anti-GST-HRP (Sigma-Aldrich, \#A7340), anti-HA-HRP (Santa Cruz Biotechnology, \#sc-7392), anti-GFP (Santa Cruz Biotechnology, \#sc9996), anti-p-Akt substrate (Cell Signaling Technology, \#9611s), antiAkt (Cell Signaling Technology, \#4691s), anti-p-Akt S473 (Cell Signaling Technology, \#4060), anti- $\beta$-actin (Abcam, \#ab8227), anti- $\alpha$-syn (Santa Cruz Biotechnology, \#sc-69977), anti-p- $\alpha$-syn S129 (Santa Cruz Biotechnology, \#sc-135638), anti-TrkB (R\&D Systems, MAB397), anti-p-TrkB pY816 (K.Y. laboratory), anti-BDNF (Novus Biologicals, \#NBP242215), anti-TH (Cell Signaling Technology, \#2792s), anti-NQO1 (Cell Signaling Technology, \#3187s; and Santa Cruz Biotechnology, \#sc32793), anti-asparagine endopeptidase (AEP) 6E3, a gift from Dr. Colin Watts (Department of Immunobiology, Division of Cell Signaling and Immunology, College of Life Sciences, University of Dundee, Dundee, United Kingdom), anti-p-C/EBP $\beta$ (Cell Signaling Technology, \#3084s), anti-C/EBP $\beta$ (Santa Cruz Biotechnology, sc-7962), anti-nuclear erythroid 2-related factor 2 (Nrf2) (Santa Cruz Biotechnology, sc-722), antiPINK1 (Cell Signaling Technology, \#6946s), anti-NQO1 T128 (K.Y. laboratory), and anti- $\alpha$-syn N103 (K.Y. laboratory). $\left[\gamma^{-}{ }^{32} \mathrm{P}\right]$-ATP (PerkinElmer, \#BLU002250UC), DAPI (Sigma-Aldrich, \#D9542), Protein A/G PLUS-Agarose (Santa Cruz Biotechnology, \#sc-200), rBDNF (PeproTech, \#450-02), streptavidin AlexaFluor-594 conjugate (Thermo Fisher Scientific, \#S11227), GSH Sepharose 4B (GE Healthcare, \#17075601), NQO1 p-T128 peptide antigen (Covance), wortmannin (EMD Millipore, \#681675), LY294002 (Sigma-Aldrich, \#L9908), PD98059 (Sigma-Aldrich, \#P215), Akt i-1/2 (Sigma-Aldrich, \#A6730), QuikChange Lightning Site-Directed Mutagenesis Kit (Agilent Technologies, \#210518), and EZ-Link Sulfo-NHS-LC-Biotinylation Kit (Thermo Fisher Scientific, \#21435).

Mice, cell lines, primary cultured rat neurons, and human tissue samples. WT C57BL/6J mice and human $\alpha$-synuclein transgenic (SNCA)-Tg mice regardless of gender were purchased from the Jackson Laboratory (000664 and 023837). All rats were purchased from The Jackson Laboratory. Animal care and handling were performed according to National Institutes of Health animal care guidelines and Declaration of Helsinki and guidelines of Emory University School of Medicine. The protocol was reviewed and approved by Emory Institutional Animal Care and Use Committee. HEK293 cells were cultured in high-glucose DMEM supplemented with $10 \% \mathrm{FBS}$ and penicillin (100 units/ml)/streptomycin (100 $\mu \mathrm{g} / \mathrm{ml}$ ) (all from Hyclone). SH-SY5Y Trk-Null and SH-SY5Y-TrkB (BR6) cells were cultured in DMEM/F12 added with 10\% FBS and penicillin $(100$ units $/ \mathrm{ml}) /$ streptomycin $(100 \mu \mathrm{g} / \mathrm{ml})$. Primary cortical neurons were cultured as previously described (Zhang et al., 2014). Cell cultures were maintained at $37^{\circ} \mathrm{C}$ in an incubator with humidified atmosphere of $5 \% \mathrm{CO}_{2}$. Postmortem brain samples were dissected from frozen brains of PD, dementia with Lewy bodies (DLB), and aged-match nondemented controls from Emory Alzheimer's Disease Research Center. The study was approved by the Biospecimen Committee of Emory University School of Medicine. PD and DLB cases were clinically diagnosed and neuropathologically confirmed. Informed consent was obtained from the subjects.

Transfection and infection of the cells. All of plasmids and siRNAs were purchased from Addgene and Santa Cruz Biotechnology, respectively. PINK1 and Parkin constructs were recently described (Geisler et al., 2010). HEK293 transfection was performed using Lipofectamine 2000 (Invitrogen, \#11668019). SH-Y5Y and BR6 transfection were performed using Lipofectamine 3000 (Invitrogen, \#L3000075) according to the manufacturer's protocol with opti-MEM (Invitrogen, \#31985070) as a transfection solution. Transfected cells were incubated at $37^{\circ} \mathrm{C}$ for $48 \mathrm{~h}$ and then harvested for further experiments.

Western blotting. To prepare whole-cell lysates, the human tissue samples, mouse brain, or cultured cells were lysed in lysis buffer (50 M Tris$\mathrm{HCl}, \mathrm{pH}$ 7.5, $150 \mathrm{M} \mathrm{NaCl}, 1 \mathrm{~m}$ EDTA, 0.5\% Triton X-100, 50 м NaF, $10 \mathrm{M}$ sodium pyrophosphate, $10 \mathrm{M}$ sodium $\beta$-glycerophosphate, $1 \mathrm{M} \mathrm{Na}_{3} \mathrm{VO}_{4}$, supplemented with a mixture of protease inhibitors) on ice for $15 \mathrm{~min}$. 
Cell lysates were centrifuged at $15,000 \times g$ for $15 \mathrm{~min}$ at $4^{\circ} \mathrm{C}$, and then the supernatant was collected. Protein concentration was measured by Bradford protein assay (Bio-Rad, \#5000006). Equally amount of protein $(20-50 \mu \mathrm{g})$ was loading for blotting with specific antibodies indicated.

Immunoprecipitation. The cultured cells, mouse brain tissue, or human tissue samples were lysed in lysis buffer and centrifuged for $15 \mathrm{~min}$ at $15,000 \times g$ at $4^{\circ} \mathrm{C}$. The supernatant was incubated with GSH Sepharose 4B Beads for GST target protein, or with indicated antibody and protein A/G-agarose together overnight at $4^{\circ} \mathrm{C}$. After extensive washing, the bound proteins were detached from the beads by boiling in sample buffer for $10 \mathrm{~min}$ and then subjected to Western blot analyses.

Immunostaining. Free-floating mouse brain sections or paraffinembedded human brain sections were treated with $0.3 \% \mathrm{H}_{2} \mathrm{O}_{2}$ for 10 min. For immunofluorescence staining, sections were washed with PBS for three times and blocked in blocking buffer (1\% BSA, $0.3 \%$ Triton $\mathrm{X}-100$, and $10 \%$ goat serum in PBS) for $1 \mathrm{~h}$, followed by overnight incubation with primary antibody at $4^{\circ} \mathrm{C}$. After washing with carrier buffer (1\% BSA, $0.3 \%$ Triton X-100, and $1 \%$ goat serum in PBS), incubated with AlexaFluor-555-conjugated anti-rabbit or anti-mouse IgG for $2 \mathrm{~h}$ at room temperature, followed by DAPI staining for $5 \mathrm{~min}$. Immunochemistry staining was performed according to the manufacturer's instructions (Invitrogen, \#95-6143). Briefly, free-floating 25- $\mu \mathrm{m}$-thick serial sections were treated with $0.3 \%$ hydrogen peroxide for $10 \mathrm{~min}$. Then, sections were rinsed three times with PBS and blocked in Reagent 1A for $10 \mathrm{~min}$ followed by incubation with primary antibodies at $4^{\circ} \mathrm{C}$ overnight. After washing with PBS, sections incubated with a biotinylated second antibody (Reagent 1B) followed by the conjugate enzyme (Reagent 2 ) for each $10 \mathrm{~min}$. Then, a chromogen AEC single solution was used to develop the signals.

NQO1 activity assay. For studies with recombinant enzyme preparations, human GST-NQO1 WT and GST-NQO1 S40A/T128A proteins were expressed in HEK293 cells and purified using GSH Sepharose 4B Beads. NQO1 activity was measured as the rate of dicoumarol inhibiting DCPIP reduction essentially as described previously (Dehn et al., 2003). Briefly, the reaction mixture in final volume of $200 \mu \mathrm{l}$ contained $25 \mathrm{M}$ Tris, pH 7.4, $0.2 \mathrm{mg} / \mathrm{ml} \mathrm{BSA,} \mathrm{pH} \mathrm{7.4,} 5 \mu \mathrm{M}$ FAD, $200 \mu \mathrm{M}$ NADH, $0.01 \%$ Tween $20(\mathrm{w} / \mathrm{v})$, and $20 \mu \mathrm{M}$ DCPIP. Enzyme activity was determined by monitoring the enzyme-dependent decrease in absorbance at $600 \mathrm{~nm}$ with the presence or absence of $20 \mu \mathrm{M}$ dicumarol as an inhibitor.

$A E P$ activity assay. Brain tissue homogenates $(30 \mu \mathrm{g})$ were incubated in $200 \mu \mathrm{l}$ reaction buffer containing $20 \mathrm{M}$ citric acid, $60 \mathrm{M} \mathrm{Na}_{2} \mathrm{HPO}_{4}, 1 \mathrm{M}$ DTT, $1 \mathrm{~m}$ EDTA, and 0.1\% CHAPS with pH 6.0, added $10 \mu \mathrm{M}$ AEP substrate Z-Ala-Ala-Asn-AMC (Bachem) inside. AMC released by AEP cleavage was quantified by measuring at $460 \mathrm{~nm}$ in a fluorescence plate reader at $37^{\circ} \mathrm{C}$ in kinetic mode.

Real-time PCR. Levels of mRNA were analyzed by qRT-PCR. RNA was isolated by Trizol (Invitrogen, \#15596018). Reverse transcription was performed with SuperScriptIII reverse transcriptase (Invitrogen, $\# 18080085$ ), and gene-specific primers and probes were designed and bought from TaqMan. All real-time PCRs were performed using the ABI 7500-Fast Real-Time PCR System and TaqMan Universal Master Mix Kit (Invitrogen, \#4304473). The relative quantification of gene expression was calculated as $2^{-\Delta \Delta \mathrm{Ct}}$ method. All of the data points were collected with at least duplicated wells, and each experiment was repeated at least three times.

Generation of antibodies specific for Akt-phosphorylated NQO1 (antiNQO1 T128). Two rabbits were immunized with the peptide Ac-EFAY(pT)YAAMYDKCKK-amide. The rabbits received booster injections 4 times with the immunizing peptide with 3 week intervals between injections. The collected antiserum was affinity purified by chromatography with the immunizing peptide and then was adsorbed to a peptide spanning the phosphorylated site (Ac-EFAYTYAAMYDK CKK-amide).

Akt in vitro kinase assay. Human recombinant GST and Tau/GST-Tau proteins were expressed in HEK293 cells and purified using GSH Sepharose 4B Beads or by immunoprecipitation. NQO1 protein was purchased from Sigma-Aldrich (\#D1315). Recombinant human GST-tagged Akt protein was purchased from Cell Sciences (\#CRA004A). Substrates were incubated with Akt in a kinase reaction buffer ( $25 \mathrm{M}$ Tris- $\mathrm{HCl}, \mathrm{pH} 7.5,10$
$\mathrm{M} \mathrm{MgCl}_{2}, 5 \mathrm{M} \beta$-glycerophosphate, $2 \mathrm{M} \mathrm{DTT}$, and $\left.0.1 \mathrm{M} \mathrm{Na}_{3} \mathrm{VO}_{4}\right)$ containing fresh $20 \mu \mathrm{M}$ ATP, $0.1 \mathrm{mg} / \mathrm{ml}$ heparin, and $1.5 \mu \mathrm{Ci}$ of $\left[\gamma^{-}{ }^{32} \mathrm{P}\right]$ for $2 \mathrm{~h}$ at $30^{\circ} \mathrm{C}$. Reactions were then separated in SDS-PAGE and analyzed by autoradiography.

Identification of phosphorylation sites on NQO1 by liquid chromatographytandem mass spectrometry. The experiment was performed based on an optimized protocol (Pagala et al., 2015). The NQO1 gel band was digested with trypsin, and the resulting peptides were analyzed by a nanoscale reverse phase liquid chromatography coupled with an LTQ Orbitrap Elite mass spectrometer (Thermo Fisher Scientific). The HPLC was performed on a $75 \mu \mathrm{m}$ inner diameter $\times 40 \mathrm{~cm}$ column $(1.9 \mu \mathrm{m} \mathrm{C18}$ resin, Dr. Maisch, HPLC) with a 90 min gradient of $15 \%-50 \%$ B (solvent A: $0.1 \%$ formic acid; solvent B: $0.1 \%$ formic acid, $70 \%$ acetonitrile; flow rate of $260 \mathrm{nl} / \mathrm{min}$ ). The mass spectrometer was operated in datadependent mode with MS1 survey scans in the Orbitrap $\left(1 \times 10^{6}\right.$ target value; 240,000 resolution; $300-1400 \mathrm{Th}, 1.6 \mathrm{Th}$ isolation window) followed by MS/MS scans analyzed in the ion trap (top 20, CID fragmentation; normalized collision energy of 35;2 $\times 10^{4}$ target value). Dynamic exclusion duration was set to $12 \mathrm{~s}$. Maximum injection times were set to $50 \mathrm{~ms}$ for all MS1 scans and $100 \mathrm{~ms}$ for MS/MS scans. Acquired MS/MS raw files were converted into mzXML format and searched by JUMP algorithm against a composite target/decoy database to estimate false discovery rate (Wang et al., 2014). Searching parameters included mass tolerance of precursor ions $( \pm 6 \mathrm{ppm})$ and product ion $( \pm 0.5 \mathrm{Da})$, tryptic restriction, dynamic mass shifts for oxidized Met (15.9949) and phosphorylated Ser, Thr, and Tyr (79.96633), three maximal modification sites, two maximal missed cleavages, as well as only $b$ and $y$ ions counted. Assigned peptides were grouped by charge state and then filtered by matching scores (XCorr and $\Delta \mathrm{Cn}$ ) to reduce the protein false discovery rate to $\sim 1 \%$.

ROS detection. Chloromethyl- $\mathrm{H}_{2}$ DCFDA (CM- $\mathrm{H}_{2}$ DCFDA) was used as a probe for the assay of ROS content. In brief, removing cells from growth media via pipetting and then resuspended cells in prewarmed PBS buffer containing the probe to provide a final working concentration of $5 \mu \mathrm{M}$ at $37^{\circ} \mathrm{C}$ for $30 \mathrm{~min}$. The reaction was terminated by chilling the reaction mixture in ice. Formation of the oxidized fluorescent derivative (DCF) was monitored at EX/EM of $485 / 528 \mathrm{~nm}$ using a fluorescence spectrophotometer. All procedures were performed in the dark 96-well plates with blanks containing only $\mathrm{CM}-\mathrm{H}_{2} \mathrm{DCFDA}$ as the measurement of autofluorescence.

Protein carbonyl assay. Protein carbonyls were measured for cell lysates or brain homogenates using 2,4-dinitrophenylhydrazine derivatization according to the manufacturer's instruction of Protein Carbonyl Assay Kit (Abcam, \#ab126287).

GSH/GSSG analysis. GSH/GSSG ratio in cells or brain samples were determined using GSH/GSSG Ratio Detection Assay Kit (FluorometricGreen; Abcam, \#ab138881) according to the manufacturer's guidelines. In brief, a serial dilution of GSH and GSSG stock standards were prepared as standards; the whole-cell lysates or brain SN lysates were diluted to 1:80 for GSH analysis. A one-step fluorimetric reaction of samples with respective assay buffer, and probes were incubated for $60 \mathrm{~min}$ protected from light. Then, fluorescence intensity was monitored at EX/EM of 490/520 nm. GSH was calculated from the standard cure and GSSG $=$ (Total GSH - GSH)/2.

Stereotaxic injection of the virus into the $S N$. The coding sequence of NQO1 WT or NQO1 S40A/T128A was inserted into pFCGW-N1 lentiviral vectors (CMV promoter, GFP tag). The virus was packaged by Viral Vector Core of Emory University. Three-month-old SNCA mice regardless of gender were anesthetized with isoflurane (Piramal Healthcare). Meloxicam (2 mg/kg) was injected subcutaneously as an analgesic (Loxicon, Norbrook). Bilateral intracerebral injection of lentivirus GFP, lentivirus NQO1 WT (titer: $5 \times 10^{9} \mathrm{IU} / \mathrm{ml}$ ), and lentivirus NQO1 S40A/ T128A (titer: $4 \times 10^{9} \mathrm{IU} / \mathrm{ml}$ ) were performed stereotactically at coordinates AP $-3.1 \mathrm{~mm}$ and $\mathrm{ML}-1.2 \mathrm{~mm}$ relative to bregma, and $\mathrm{DV}$ $-4.3 \mathrm{~mm}$ from the dural surface. A volume of $2 \mu \mathrm{l}$ of viral suspension was injected into each point using $10 \mu \mathrm{l}$ Hamilton syringe with a fixed needle ant a rate of $0.25 \mu \mathrm{l} / \mathrm{min}$. Remained the needle in site for $5 \mathrm{~min}$ before it was removed out slowly (over $2 \mathrm{~min}$ ). The mice were placed on a heating pad until they began to recover from the surgery. 
MPTP injection and behavioral test. Mice were injected a daily intraperitoneal injection of MPTP $(30 \mathrm{mg} / \mathrm{kg})$ or saline treatment for $5 \mathrm{~d}$ after virus injection at 2 months. Motor impairments were tested 1 week after MPTP treatment with rotarod tests and grid tests. In the rotarod tests, mice were trained for $2 \mathrm{~min}$ at a speed of $4 \mathrm{rpm}$ and then performed eight trials for a maximum of 5 min with increasing speed starting from 4 to 40 rpm. The fall-off time was recorded. For inverted grid tests, mice were placed in the center of a $30 \times 30 \mathrm{~cm}$ screen with $1-\mathrm{cm}$-wide mesh. The screen was inverted head-over-tail and placed on supports $40 \mathrm{~cm}$ above an open cage with deep bedding. Mice were timed until they released their grip or remained for $60 \mathrm{~s}$.

Experimental design and statistical analysis. The primary aim of this study was to explore the molecular mechanism dictating NQO1 expression oscillation in neurodegenerative disease. All completed experiments followed the principle of from in vitro to in vivo study to solidly confirm our findings. After the proteomic analysis to indicate the phosphorylation sites, three NQO1 mutant plasmids (S40A, T128A, and S40A/ T128A) and innovative antibody NQO1-pT128 were made to perform the cell experiments. Both male and female mice, WT, and $\alpha$-SNCA transgenic mice were used in the animal experiments. Eight to 10 mice were performed for behavioral tests, and 4-6 samples from each group were analyzed in staining experiments. Statistical analysis was performed with Prism 7.0 (GraphPad). All data were expressed as mean \pm SEM from three or more independent experiments. Histological data were analyzed using either Student's $t$ test (two-group comparison) or one-way ANOVA or two-way ANOVA. The threshold for significance for all experiments was set at $p<0.05$, and smaller $p$ values were represented as $p<0.01$ and $p<0.001$.

\section{Results}

\section{Akt phosphorylates NQO1 on S40 and T128 residues}

To assess whether Akt phosphorylates NQO1, we conducted an in vitro Akt phosphorylation assay with commercial recombinant NQO1 proteins in the presence of $\left[\gamma_{-}{ }^{32} \mathrm{P}\right]$-ATP. Autoradiography showed that both positive control Tau and NQO1 were evidently phosphorylated by Akt, whereas GST-negative control was not. Akt was highly auto-phosphorylated (Fig. 1A, top). To map the phosphorylation sites on NQO1, we performed proteomic analysis with the in vitro phosphorylated NQO1 and found that both S40 and T128 residues were phosphorylated by Akt (Fig. $1 B, C)$. To confirm phosphorylation of these two specific sites, both residues were mutated to alanine and used for in vitro phosphorylation assays. Compared with WT NQO1, mutation of either of these two residues reduced overall phosphorylation levels. Phosphorylation was completely abolished for the S40A/T128A double mutant (Fig. 1D). Next, we developed a rabbit polyclonal phospho-T128 NQO1-specific antibody. The in vitro phosphorylated NQO1 was not only validated by p-Akt substrate antibody that nonspecifically recognizes all of Akt phosphorylation motifs, but also visualized by p-T128 antibody (Fig. 1E). To test whether Akt phosphorylates NQO1 in intact cells, we used TrkB-stably transfected dopaminergic SH-SY5Y cells (BR6 cells). Knockdown of Akt strongly diminished p-NQO1 T128 signals in BR6 cells (Fig. 1-1A, available at https://doi.org/ 10.1523/JNEUROSCI.0625-19.2019.f1-1), suggesting that Akt may indeed phosphorylate NQO1. To test this antibody's specificity, we found that p-NQO1 T128 antibody only selectively recognized WT NQO1, but not the S40A/T128A double mutant, in the transfected cells. Moreover, this activity was blocked by preincubation with the phospho-peptide antigen, indicating the specificity of the antibody (Fig. 1-1 B, available at https://doi.org/ 10.1523/JNEUROSCI.0625-19.2019.f1-1). Immunohistochemistry staining with $\alpha$-SNCA transgenic PD mouse brain sections revealed that p-NQO1 signals were abrogated by the antigen peptide, but not nonspecific peptide (Fig. 1-1C, available at https://
doi.org/10.1523/JNEUROSCI.0625-19.2019.f1-1), indicating that this phospho-antibody specifically recognizes p-NQO1 T128. To further explore whether this phosphorylation occurs in the PD mouse models, we analyzed p-NQO1 T128 signals in different ages of human $\alpha$-SNCA transgenic mice. Immunoblotting showed that p-Akt activities tightly correlated with p-NQO1 T128 signals, and they were gradually reduced with advanced age, underscoring that Akt is responsible for phosphorylating NQO1 in PD mouse models. Notably, NQO1 levels peaked at 7-11 months of age and progressively declined afterward, so was another antioxidative-related transcription factor FOXO3a. Noticeably, Nrf2 and PINK1 expression levels were also reduced in an age-dependent manner, partially in alignment with NQO1 levels in $\alpha$-SNCA transgenic mice (Fig. $1 F$ ). It is worth noting that the oxidative stress was age-dependently augmented in $\alpha$-SNCA mice (Fig. 1-1 D, E, available at https://doi.org/10.1523/ JNEUROSCI.0625-19.2019.f1-1). Thus, Akt phosphorylates NQO1 T128 residue in PD mouse model.

\section{Akt associates with NQO1 in a phosphorylation-dependent manner}

To examine whether Akt associates with NQO1, we performed GST pulldown assay from cells cotransfected with both GSTNQO1 and various HA-tagged Akt constructs. Interestingly, Akt-CA (constitutively active) displayed the strongest binding activity, followed by WT Akt, whereas Akt-KD (kinase-dead) failed to associate with NQO1. The association patterns correlated well with p-NQO1 levels, suggesting that the interaction between NQO1 and Akt might be mediated by its phosphorylation status (Fig. 2A, top). The relative binding affinities and p-NQO1 levels were quantified (Fig. 2A, bottom). To test whether $\mathrm{p}-\mathrm{NQO} 1$ status indeed regulates the complex formation, we used phospho-dead mutants and found that S40A or T128A diminished binding between Akt and NQO1. It is worth noting that T128A exhibited much weaker binding compared with $\mathrm{S} 40 \mathrm{~A}$, and the interaction was completely abrogated when both residues were mutated into A, supporting that NQO1 phosphorylation is required for Akt/NQO1 association with p-T128 being more important than p-S40 (Fig. 2B).

To further examine whether their interaction is p-NQO1dependent, we treated primary cortical neurons with BDNF in the presence of various inhibitors. Compared with DMSO control, BDNF treatment enhanced the interaction, but inhibition of the PI3K/Akt pathway with wortmannin, Akt i-1/2, or LY294002, but not MEK1, with PD98059 evidently decreased the interaction between NQO1 and Akt. As expected, both p-Akt and p-NQO1 signals were strongly repressed by the inhibitors, fitting with the binding activities between NQO1 and Akt (Fig. 2C). We made similar observations with endogenous Akt and NQO1 upon EGF treatment of SH-SY5Y cells. Remarkably, total NQO1 levels inversely correlated with p-Akt and p-NQO1 levels (Fig. 2-1 A, available at https://doi.org/10.1523/JNEUROSCI.0625-19.2019. f2-1). Thus, the interaction between Akt and NQO1 is p-T128dependent. To further explore the association of these two proteins, we conducted immunofluorescent staining with $\mathrm{PD}$ patients' SN and age-matched healthy controls. Both Akt/pNQO1 and p-Akt/p-NQO1 were strongly colocalized in the cytoplasm. Notably, both p-NQO1 and p-Akt levels were markedly reduced in PD brains compared with healthy controls (Fig. $2 D-$ G). Similar to patients, p-NQO1/Akt and Akt/p-NQO1 were also strongly colocalized in WT mice brains, and both were potently reduced after MPTP treatment. In agreement with these findings, we found that chronic MPTP treatment repressed both p-Akt and 
A

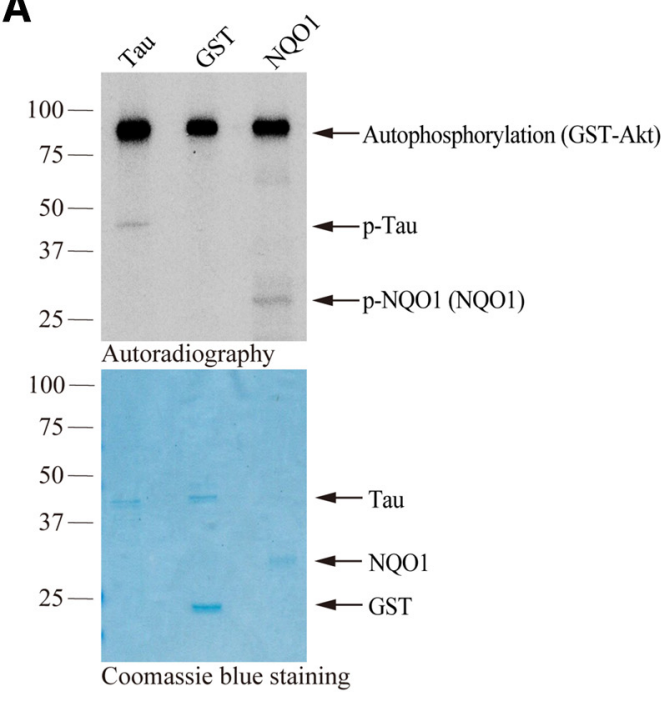

B

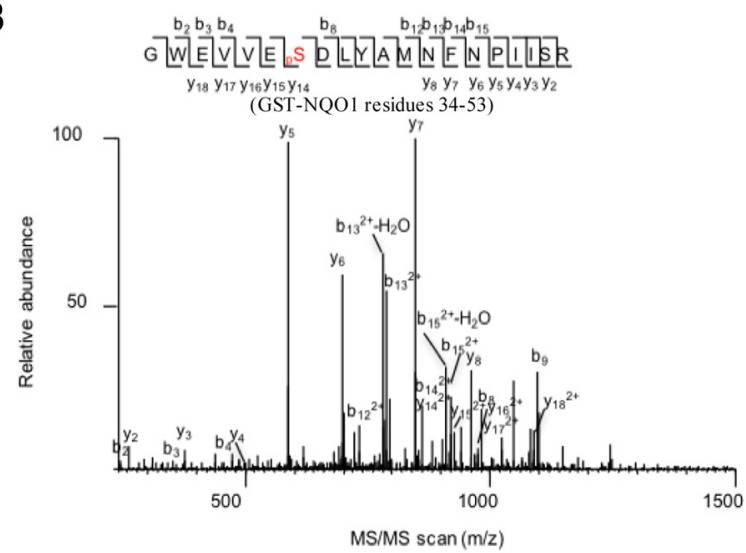

D

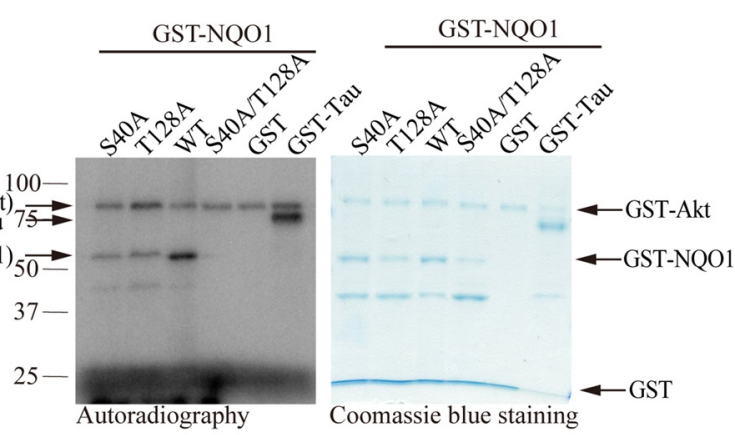

Autophosphorylation (GST-Akt) $\underset{7 \rightarrow}{100-}$ $b_{3} \quad b_{5} b_{6} b_{7} b_{8} b_{9} b_{10} b_{11} b_{12} b_{13}$

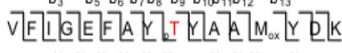
$y_{13} y_{12} y_{11} y_{10} y_{9} y_{8} \quad y_{6} y_{5} y_{4} \quad y_{5}$

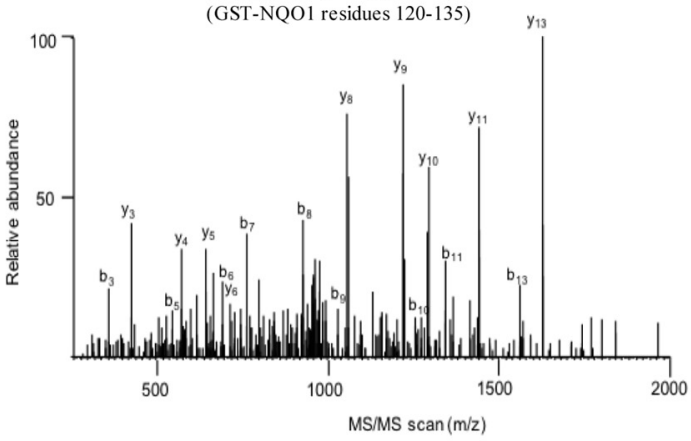

F

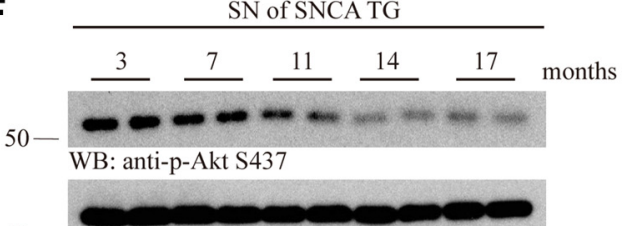

E

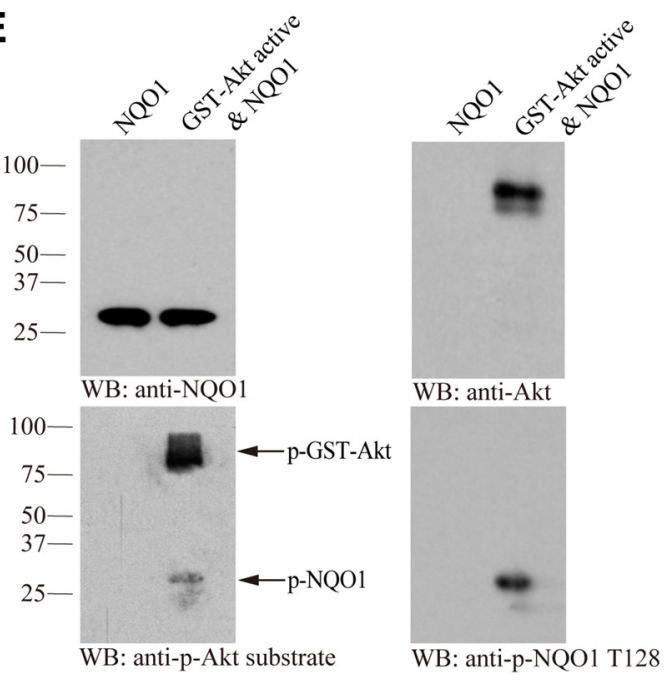

$50-$ WB: anti-Akt

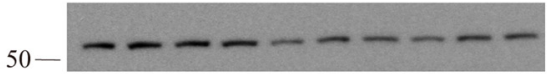

WB: anti-Nrf2

$50-$

WB: anti-PINK1
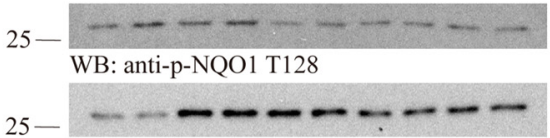

WB: anti-NQO1
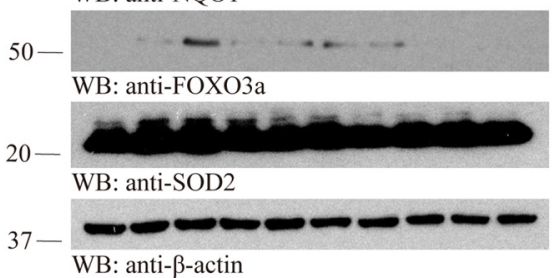

Figure 1. Akt phosphorylates NQ01 on S40 and T128 residues. $A$, In vitro kinase assay. Recombinant NQ01 proteins were incubated with GST-Akt in the presence of [ $\gamma^{-}{ }^{32}$ P]-ATP. Tau and GST alone were used as positive and negative controls, respectively. Top, The reaction mixture was resolved on 10\% SDS-PAGE and analyzed by autoradiography. Bottom, Coomassie Blue staining of protein inputs. B, C, MS/MS spectra showing the phosphorylation of NQ01 on residues S40 and T128 in GST-NQ01 purified from HEK293 cells cotransfected with GST-NQ01 and HA-Akt plasmids. D, S40 and T128 are Akt phosphorylation sites on NQ01. S40A (S to A, unphosphorylatable mutant) or T128A (T to A, unphosphorylatable mutant) mutation decreased the NQ01 phosphorylation, whereas phosphorylation was completely blocked in the double-mutant $\mathrm{S} 40 \mathrm{~A} / \mathrm{T} 128 \mathrm{~A}$ (left). Right, The protein inputs. $\boldsymbol{E}$, Western blot analysis of the reaction products of in vitro Akt kinase assay with NQ01 recombinant proteins using p-Akt substrate antibody and p-T128 antibody. Validation of the specificity of anti-p-NQ01 T128 can be found in Figure 1-1A-C (available at https://doi.org/ 10.1523/JNEUROSCI.0625-19.2019.f1-1). F, Phosphorylated NQ01 T128 expression levels tightly correlate with p-Akt activity in SN of $\alpha$-SNCA mouse brains. The expression levels of Nrf2, PINK1, NQ01, and FOX03a and the oxidative stress (see Figure 1-1 D, E, available at https://doi.org/10.1523/JNEUROSCI.0625-19.2019.f1-1) changed with age increase. Blot data are representative of three independent experiments. 
A
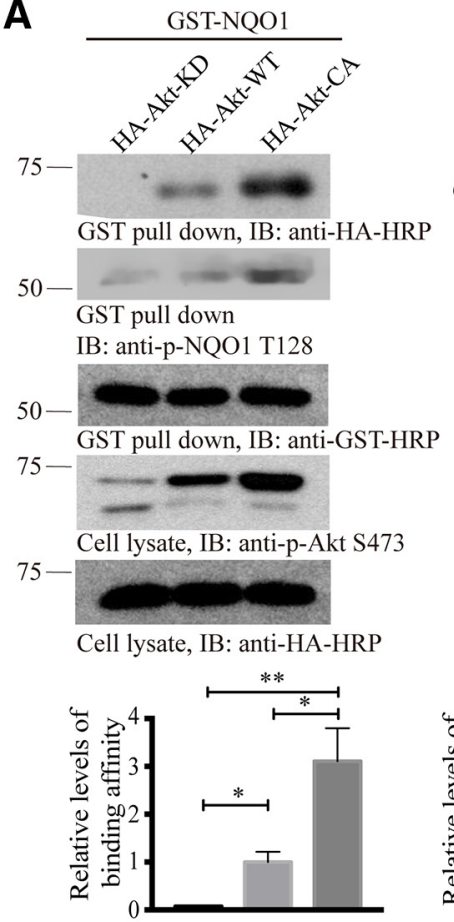

D
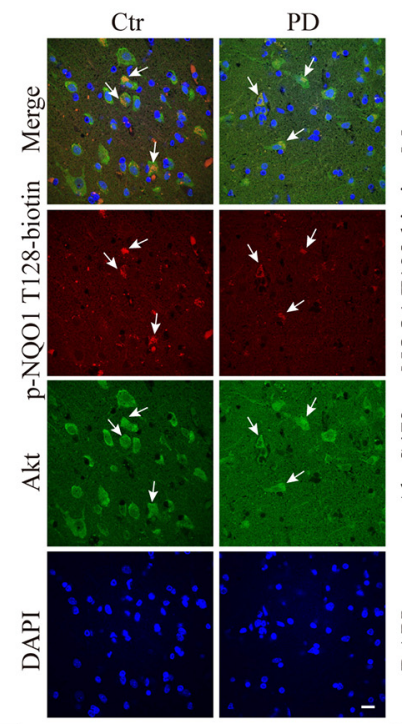

F

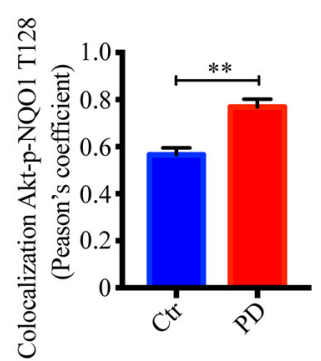

B

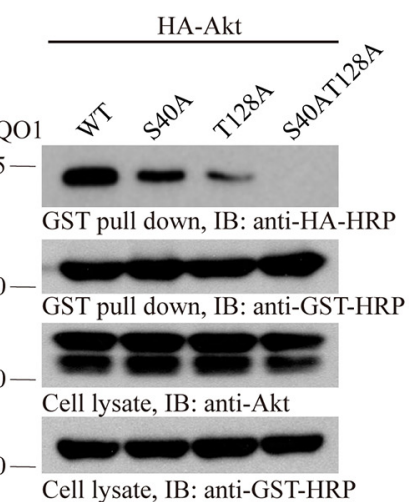

C Rat Cortical Neuron

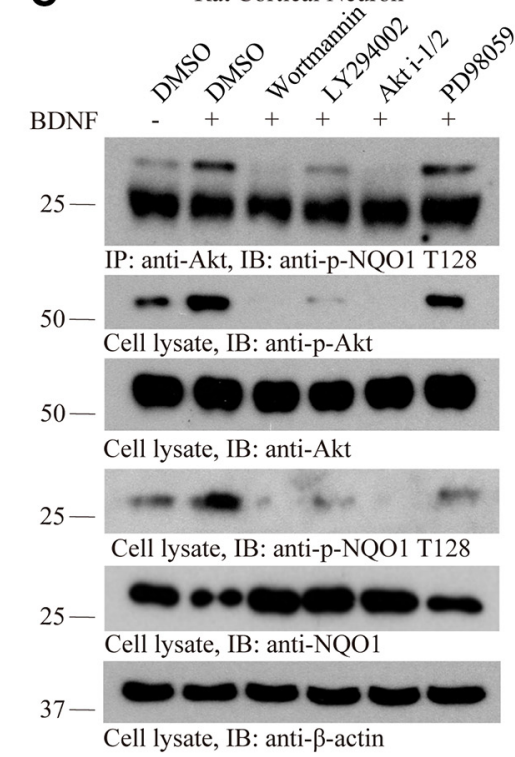

$\mathbf{H}$

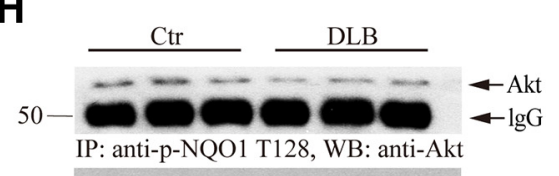

E

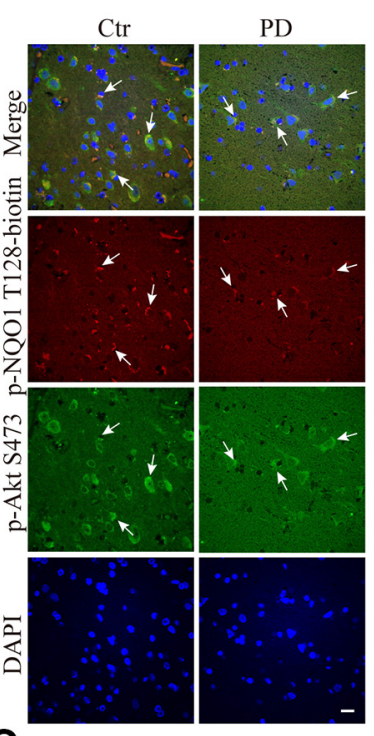

G

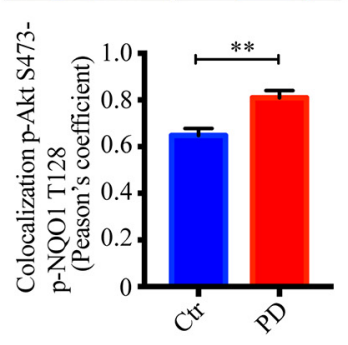

- $-\cdots$

WB: anti-BDNF

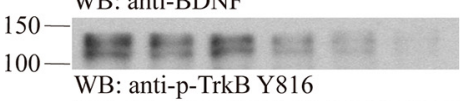

150 - $100-C$

WB: anti-TrkB

$50-\frac{-}{\text { WB: anti-p-Akt S473 }}-\cdots+\cdots$

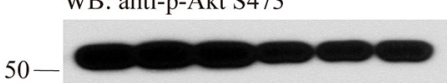

WB: anti-Akt

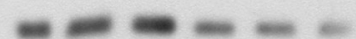

WB: anti-p-NQO1 T128

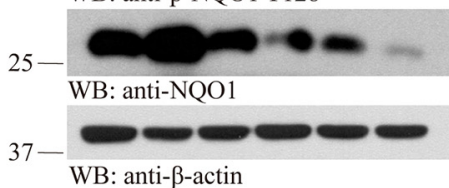

I

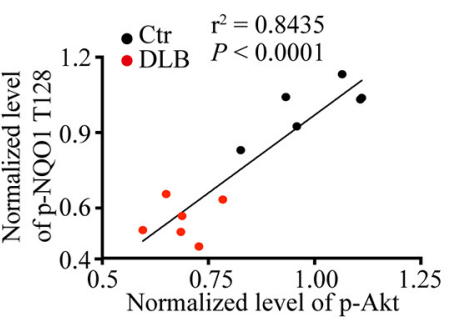

Figure 2. Akt associates with NQ01 in a phosphorylation-dependent manner. A, Akt kinase activity is required for its association with NQ01. Mammalian GST-NQ01 was cotransfected with HA-Akt KD, WT, or CA into HEK293 cells. After $48 \mathrm{~h}$, GST-NQ01 was pulled down with GSH beads and subjected to Western blot analysis with anti-HA and p-NQ01 T128 antibodies, which showed Akt WT and CA, but not KD, robustly bound to NQ01 (top) with the same pattern of p-NQ01 T128 (second panel). Bottom, Quantification of relative binding affinity and p-NQ01 T128 levels ( $n=3$ ). $\boldsymbol{B}$, Western blot analysis of phosphorylation level of WT NQ01 and mutated ones after cotransfection with Akt. Mutation of T128 into A abolished Akt phosphorylation of NQ01. C, Akt associates with NQ01 in primary neurons. The interaction was tested in primary rat neurons pretreated with PI(3)K inhibitors or a MEK inhibitor for $30 \mathrm{~min}$ and followed by BDNF treatment (100 ng/ml, $30 \mathrm{~min}$ ). The total levels of NQ01 were inversely correlated with its p-NQ01 status (fourth and fifth panels). Similar observations in SH-SYSY cells can be found in Figure 2-1 A (available at https://doi.org/10.1523/ JNEUROSCI.0625-19.2019.f2-1).D, E, Immunofluorescent staining of Akt/p-NQ01 T128 and p-Akt S473/p-NQ01 T128 in human PD SN compared with age-matched healthy control. Confocal images show colocalization between p-Akt/p-NQ01. Although both of their activities were reduced in PD compared with healthy control, colocalization was increased in PD. (Figure legend continues.) 
p-NQO1 levels in WT mice compared with vehicle control with a tight correlation. Remarkably, NQO1, but not Akt, total levels were decreased upon MPTP treatment (Fig. 2-1B-F, available at https://doi.org/10.1523/JNEUROSCI.0625-19.2019.f2-1). The same phenomena occurred in human $\alpha$-SNCA transgenic PD mouse model upon MPTP treatment (Fig. 2-1G-K, available at https://doi.org/10.1523/JNEUROSCI.0625-19.2019.f2-1). To further investigate the biochemical events in human DLB patients, another synucleinopathy, we found that BDNF was evidently reduced in DLB samples compared with control brains. Accordingly, both $\mathrm{p}$-TrkB and its downstream effector $\mathrm{p}$-Akt signals were decreased in DLB patient brains. In alignment with these findings, both p-NQO1 and total NQO1 were prominently attenuated in DLB samples versus controls. Consequently, coimmunoprecipitation assay demonstrated that the association between p-NQO1 and Akt was decreased (Fig. 2H,I). Therefore, the association between Akt and NQO1 is phosphorylation-dependent, and the phosphorylation signals are diminished in PD mouse models and patient brains.

\section{Akt phosphorylation triggers NQO1 degradation}

The inverse correlation between p-Akt and NQO1 total levels suggests that Akt phosphorylation of NQO1 might trigger its protein degradation. To investigate this possibility, we used BR6 cells that were treated with BDNF and PI(3)K inhibitors. We found that NQO1 levels inversely correlated with p-Akt levels, and NQO1 levels were high when Akt was inhibited by its inhibitors, and BDNF treatment strongly decreased NQO1 protein levels (Fig. 3A). To further explore whether indeed Akt dictates NQO1 protein stability, we transfected different amounts of Akt-CA constructs into HEK293 cells, and found that NQO1 levels were gradually diminished when Akt was increased (Fig. $3 B$ ). TaqMan Real-Time PCR Assay for the specific detection and quantification (Connell and Messerschmidt, 2005) of NQO1 confirmed that Akt-CA did not affect mRNA levels of NQO1 (Fig. $3 C$ ), indicating that Akt phosphorylation of NQO1 may mediate its protein stability but not mRNA transcription.

To assess whether p-NQO1 is degraded through polyubiquitination/proteasomal pathway, we transfected HA-NQO1 into HEK293 cells, followed by EGF stimulation for different time points. Immunoprecipitation experiments showed that NQO1 was ubiquitinated in a phosphorylation-dependent manner. When p-Akt signals faded away, NQO1 phosphorylation reduced, so was its ubiquitination (Fig. 3-1A, available at https:// doi.org/10.1523/JNEUROSCI.0625-19.2019.f3-1). To determine whether this effect was triggered by Akt, we cotransfected GSTNQO1 and HA-tagged Akt constructs into HEK 293 cells. GST pulldown revealed that NQO1 was strongly polyubiquitinated in the presence of Akt-CA, and this effect was reduced in the presence of Akt-KD, coupled with p-NQO1 status (Fig. 3-1 B, available at https://doi.org/10.1523/JNEUROSCI.0625-19.2019.f3-1).

(Figure legend continued.) Scale bars, $20 \mu \mathrm{m} . \boldsymbol{F}, \mathbf{G}$, Quantification of colocalization in $\boldsymbol{D}$ and $\boldsymbol{C}(n=5$ cases per group, $p=0.0016$ in $\boldsymbol{F}, p=0.0051$ in $\boldsymbol{G}$, unpaired $t$ test, two-tailed). $\boldsymbol{H}$, Western blot analysis of phosphorylated NQ01 and Akt in DLB brains compared with agematched healthy controls. I, The expression levels of p-NQ01 T128 directly correlate with p-Akt levels in human brains. The Spearman correlation coefficient $r^{2}$ and $p$ values are shown. For the related changes of p-NQ01 and p-Akt levels in WT and $\alpha$-SNCA mice after MPTP treatment involved, see Figure 2-1B-K (available at https://doi.org/10.1523/JNEUROSCI.062519.2019.f2-1). All the Western blot data are representative of three independent experiments. Quantification data are expressed as mean \pm SEM. Student's $t$ test or one-way ANOVA with Tukey's multiple-comparisons test: ${ }^{*} p<0.05 ;{ }^{* *} p<0.01 ;{ }^{\sharp} p<0.001$.
Cotransfection assay showed that GST-NQO1 interacted with HA-ubiquitin. The total levels of GST-NQO1 were reduced in the presence of HA-ubiquitin (Fig. 3-1C, available at https://doi. org/10.1523/JNEUROSCI.0625-19.2019.f3-1), suggesting that NQO1 polyubiquitination might reduce its protein levels. To ensure that Akt phosphorylation of NQO1 dictates its polyubiquitination, we cotransfected GST-NQO1 and HA-Akt into HEK293 cells in the presence of various Akt inhibitors, and found treatment with MG132, a proteasomal inhibitor, strongly augmented NQO1 polyubiquitination, which was antagonized by Akt inhibitors. Both p-Akt and p-NQO1 activities were blocked by these inhibitors (Fig. 3D). To further interrogate the role of Akt phosphorylation of NQO1 in its polyubiquitination, we treated BR6 cells in the presence of Akt i-1/2, followed by BDNF stimulation. In the presence of MG132, BDNF treatment elicited demonstrable NQO1 polyubiquitination, which was blocked by Akt inhibitor (Fig. 3E, lanes 4 and 8), indicating that BDNFinduced Akt phosphorylates NQO1 and triggers its polyubiquitination. In HEK 293 cells cotransfected with both GST-NQO1 and HA-ubiquitin, again, EGF-elicited NQO1 polyubiquitination was also abolished in the presence of Akt inhibitor (Fig. 3-1D, available at https://doi.org/10.1523/JNEUROSCI.062519.2019.f3-1). To directly assess the role of p-T128 in dictating NQO1 polyubiquitination, we used different NQO1 mutants, and found that T128A, but not S40A, strongly blunted NQO1 polyubiquitination, and the double mutant (S40A/T128A) totally lost its polyubiquitination activities regardless of BDNF or EGF treatment (Fig. 3F; Fig. 3-1E, available at https://doi.org/ 10.1523/JNEUROSCI.0625-19.2019.f3-1). Therefore, Akt phosphorylation of NQO1 T128 regulates its polyubiquitination and subsequent degradation.

\section{Parkin acts as an E3 ubiquitin ligase for NQO1}

Parkin belongs to the "really interesting new gene" (RING) finger class of E3 ubiquitin ligases (Shimura et al., 2000), which ubiquitinates several candidate substrates and thereby targets them for proteasomal degradation. Recessive mutations in the human PARKIN gene are the most common cause of hereditary Parkinsonism (Kitada et al., 1998). To explore whether Parkin acts as an E3 ligase for mediating NQO1 ubiquitination and degradation, we conducted a cotransfection assay and found that NQO1 strongly interacted with Parkin regardless of Akt (Fig. 4-1A, available at https://doi.org/10.1523/JNEUROSCI.0625-19.2019. f4-1). Interestingly, WT NQO1, but not unphosphorylatable S40A/T128A, mutant associated with Parkin, suggesting that this interaction is p-NQO1 T128-dependent as well (Fig. 4A). Immunofluorescent costaining revealed that p-NQO1 and Parkin tightly colocalized in human healthy control brains, and these signals were significantly reduced in PD patient brains (Fig. $4 B, C)$. To ascertain the role of Parkin in regulating NQO1 ubiquitination, we cotransfected Akt WT or KD constructs with NQO1 in the presence of Parkin into HEK293 cells, followed by MG132 treatment. We found that Akt WT, but not KD, provoked NQO1 ubiquitination, which was strongly augmented by Parkin. p-NQO1 T128 signals coupled with its polyubiquitination activity (Fig. 4D). On the other hand, depletion of Parkin with a specific siRNA entirely abrogated NQO1 ubiquitination, even though NQO1 T128 was strongly phosphorylated, suggesting that Parkin is the major E3 ligase for promoting NQO1 polyubiquitination (Fig. 4E).

PINK1 accumulates on the outer membrane of damaged mitochondria, activates Parkin's E3 ubiquitin ligase activity, and recruits Parkin to dysfunctional mitochondria. Then, Parkin 


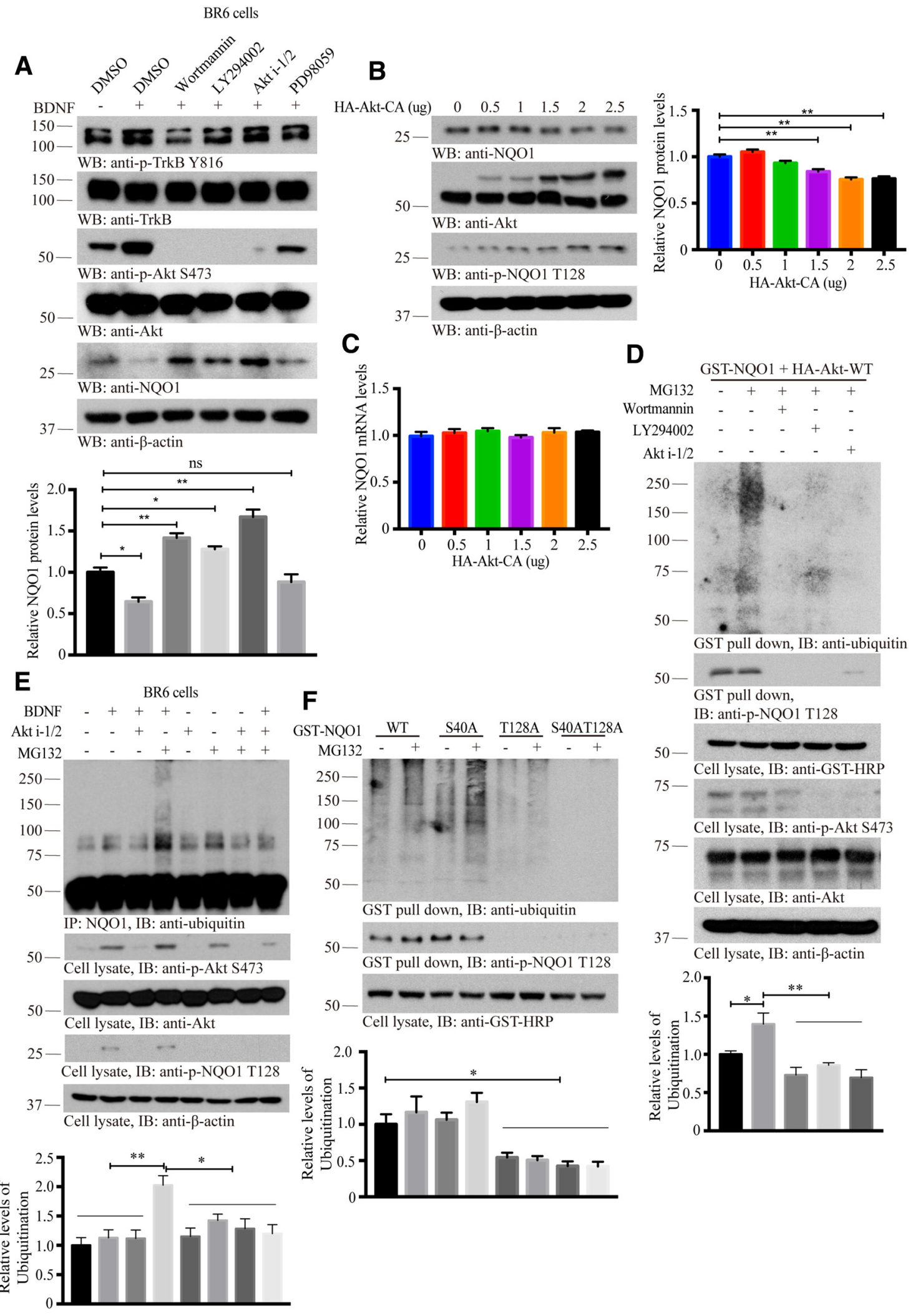

Figure 3. Akt phosphorylation triggers NQ01 degradation through ubiquitination. A, Inhibition of NQ01 phosphorylation by Akt increases its protein levels. BR6 cells were treated with PI(3)K inhibitors (wortmannin, $1 \mu \mathrm{m} ; \mathrm{LY} 294002,10 \mu \mathrm{m}$; Akt i-1/2, $10 \mu \mathrm{m}$ ) and PD98059 (10 $\mu \mathrm{M}$ ) for 30 min and followed by BDNF treatment (100 ng/ml, $30 \mathrm{~min}$ ). Western blot analyzed the cell lysates with indicated antibodies. Bottom, Quantification of relative NQ01 protein levels $(n=3)$. B, Overexpression of Akt induces NQ01 reduction. HEK293 cells were transfected with the indicated amounts of HA-Akt-CA for $48 \mathrm{~h}$. The cell lysates were subjected to Western blot analysis with indicated antibodies. Right, Quantification of relative NQ01 protein levels $(n=3$ ). C, The isolated total RNAs from samples b subjected to qRT-PCR to detect NQ01 mRNA levels ( $n=3$ per group). D, PI(3)K inhibitors abolish NQ01 ubiquitination. Mammalian GST-NQ01 was cotransfected with HA-Akt into HEK293 cells. After $36 \mathrm{~h}$, cells were pretreated with MG132 (20 $\mu \mathrm{m}, 3 \mathrm{~h})$ twice followed by PI(3)K inhibitors for $30 \mathrm{~min}$. GST-NQ01 was pulled down, and cell lysates were subjected to Western blot analysis with indicated antibodies. Bottom, Quantification of relative ubiquitination levels $(n=3)$. $\boldsymbol{E}$, Akt phosphorylation mediates NQ01 ubiquitination. BR6 cells were treated with the presence and absence of BDNF, Akt i-1/2, and MG132. NQ01 was immunoprecipitated and its ubiquitination levels were monitor by immunoblotting. Bottom, (Figure legend continues.) 
ubiquitinates outer mitochondrial membrane proteins to trigger selective autophagy (Pickrell and Youle, 2015). To assess whether PINK1 could implicate in phosphorylating NQO1 and couple it to Parkin, we cotransfected PINK1-His with GST-NQO1 WT or phospho-dead S40A/T128A mutant into dopaminergic SH-SY5Y cells, and found that Akt, but not PINK1, provoked NQO1 T128 phosphorylation, although PINK1 robustly phosphorylated Parkin. GST pulldown showed that NQO1 was prominently polyubiquitinated in the presence of Akt, but not PINK1, supporting that Akt, but not PINK1, plays a dominant role in phosphorylating NQO1 and dictates its subsequent ubiquitination (Fig. 4-1 B, available at https://doi.org/10.1523/JNEUROSCI.0625-19.2019. f4-1).

\section{Unphosphorylatable NQO1 possesses stronger antioxidative activity and exhibits higher cytoprotective effects}

To test the effect of NQO1 phosphorylation on its antioxidative enzymatic activity, we conducted in vitro NQO1 enzymatic assay and found that both WT and phospho-dead S40A/T128A mutant exhibited comparable activity, which was effectively blunted by its inhibitor Dicumarol (Fig. 5-1A, available at https://doi. org/10.1523/JNEUROSCI.0625-19.2019.f5-1). To investigate whether the phosphorylation affects NQO1's prosurvival effect, we transfected BR6 cells with GST-tagged NQO1 WT or unphosphorylatable mutant, followed by pretreatment with vehicle control or BDNF, then stimulated with $500 \mu \mathrm{M} \mathrm{MPP}{ }^{+}$. The medium was monitored with the LDH assay. We found that $\mathrm{MPP}^{+}$elicited cell death was significantly repressed by BDNF. In the absence of BDNF, transfection of NQO1 exhibited stronger cytoprotective activity than GST control regardless of WT or unphosphorylatable mutant. Nonetheless, in the presence of BDNF, S40A/T128A mutant displayed stronger cytoprotective activity than WT did (Fig. 5A). Immunoblotting analysis showed that BDNF robustly stimulated p-TrkB and p-Akt and p-NQO1 T128 signals. As expected, $\mathrm{MPP}^{+}$elicited demonstrable TH loss, which was repressed in the presence of NQO1 WT. The maximal protective effect occurred in S40A/T128A samples treated with BDNF, which was much stronger compared with WT NQO1 or vehicle control. Moreover, unphosphorylatable mutant protein concentrations were higher than WT NQO1, with lower amount of proteins in the presence of BDNF (Fig. 5B). To further assess the cytoprotective effects, we performed a TUNEL assay in the presence of $\mathrm{BDNF}$ and $\mathrm{MPP}^{+}$and found that unphosphorylatable NQO1 mutant exhibited the strongest cytoprotective activity, followed by GST-NQO1 and GST control. Consequently, TUNEL signals were the fewest in S40A/T128A-transfected cells with the strongest $\mathrm{TH}$ activities (Fig. $5 C-G$ ). The oxidative stress evaluation demonstrated that S40A/T128A mutant possessed the strongest antioxidative activities (Fig. 5-1B-D, available at https://doi.org/10.1523/JNEUROSCI.0625-19.2019.f5-1), fitting with its much higher protein stability.

\section{$\leftarrow$}

(Figure legend continued.) Quantification of relative ubiquitination levels $(n=3)$. $\boldsymbol{F}$, Akt triggers NQ01 ubiquitination-dependent on the phosphorylation of residue T128. T128mutated NQ01 (T128A and S40A/T128A) was not ubiquitinated and MG132 treatment stabilized ubiquitinated forms of WT NQ01. Bottom, Quantification of relative ubiquitination levels $(n=3)$. For the analysis of Akt triggers NQ01 ubiquitination in a phosphorylation-dependent manner involved, see Figure 3-1 (available at https://doi.org/10.1523/JNEUROSCI.062519.2019.f3-1). Data are mean \pm SEM. One-way ANOVA with Dunnett's multiplecomparisons test: ${ }^{*} p<0.05 ;{ }^{* *} p<0.01$. Western blot data are representative of three independent experiments.

\section{Unphosphorylatable NQO1 mutant protects dopaminergic neurons and rescues motor dysfunction}

To investigate the in vivo role of NQO1 phosphorylation by Akt, we injected GFP carrying lentivirus that expresses GST-tagged NQO1 WT or unphosphorylatable mutant into the SN regions of 3 -month-old $\alpha$-SNCA mice regardless of gender. The viral infection efficiency was validated by immunofluorescence colocalization analysis of GFP/TH-positive cells (Fig. 6-1 A, available at https://doi.org/10.1523/JNEUROSCI.0625-19.2019.f6-1). Two months after viral delivery, the mice were treated with MPTP (30 $\mathrm{mg} / \mathrm{kg}$, i.p.) or saline for $5 \mathrm{~d}$. We monitored dopaminergic neuron loss 1 week after MPTP treatment. Immunohistochemistry staining showed that dopaminergic neurons in $\mathrm{SN}$, and its projections in striatum were substantially diminished by MPTP in GFP control mice compared with vehicle. Again, NQO1 WT expression attenuated the loss of dopaminergic neurons. The maximal neuroprotective effects occurred to S40A/T128A mutant (Fig. 6A), which was confirmed by the quantitative analysis (Fig. $6 B, C)$. Motor behavioral tests showed that MPTP incurred significantly motor disorder in GFP control group, which was ameliorated upon NQO1 WT expression. Remarkably, in the S40A/ T128A group, MPTP treatment-elicited motor disorders were significantly less severe than the WT NQO1 group (Fig. 6D,E), supporting that unphosphorylatable NQO1 is highly neuroprotective and prevents MPTP-elicited motor dysfunctions in $\alpha$-SNCA mice. ROS analysis demonstrated that S40A/T128A mice possessed the least oxidative stress among the experimental groups, provoked by MPTP treatment, in alignment with its prominent neuroprotective activity (Fig. 6-1 $B, C$, available at https://doi.org/10.1523/JNEUROSCI.0625-19.2019.f6-1). Immunoblotting analysis revealed that comparable levels of NQO1 constructs were expressed in the mouse brains. In GFP control, MPTP treatment elicited prominent dopaminergic neuronal TH loss, which was partially alleviated by NQO1 WT expression. Dopaminergic loss was further repressed by S40A/T128A mutant (Fig. 6F, top, fourth panel).

AEP is an age-dependent protease cleaving substrates after asparagine. We have recently reported that AEP selectively cleaves $\alpha$-synuclein at N103 and promotes its aggregation and neurotoxicity in PD patient brains and PD animal models (Zhang et al., 2017). AEP is upregulated and activated by the oxidative stress exerted by MPTP (Kang et al., 2018). As expected, $\alpha$-synuclein was strongly cleaved at N103 by upregulated and activated AEP, and some of $\alpha$-synuclein was phosphorylated on S129 in GFP control mice upon MPTP. $\alpha$-Syn N103 truncation by active AEP was partially inhibited by NQO1 WT and completely repressed by unphosphorylatable mutant (Fig. 6F, fifth and sixth panels), in alignment with AEP enzymatic assay data that the least AEP activity was found in S40A/T128A mice (Fig. 6-1D, available at https://doi.org/10.1523/JNEUROSCI.062519.2019.f6-1). Immunofluorescent costaining validated that $\alpha$-Syn N103 positively correlated with AEP levels in the SN sections (Fig. 6-1E, available at https://doi.org/10.1523/JNEUROSCI.062519.2019.f6-1). MPTP provoked demonstrable p- $\alpha$-synuclein S129 in GFP control mice that were colocalized with ubiquitin, a biomarker for Lewy bodies. The signals were evidently suppressed in NQO1 WT mice and eliminated in unphosphorylatable NQO1 mutant mice (Fig. 6G). Hence, unphosphorylatable NQO1 possesses the strongest antioxidative activity, rescuing dopaminergic neurons from MPTP-induced cell death and alleviating motor impairment. 
A

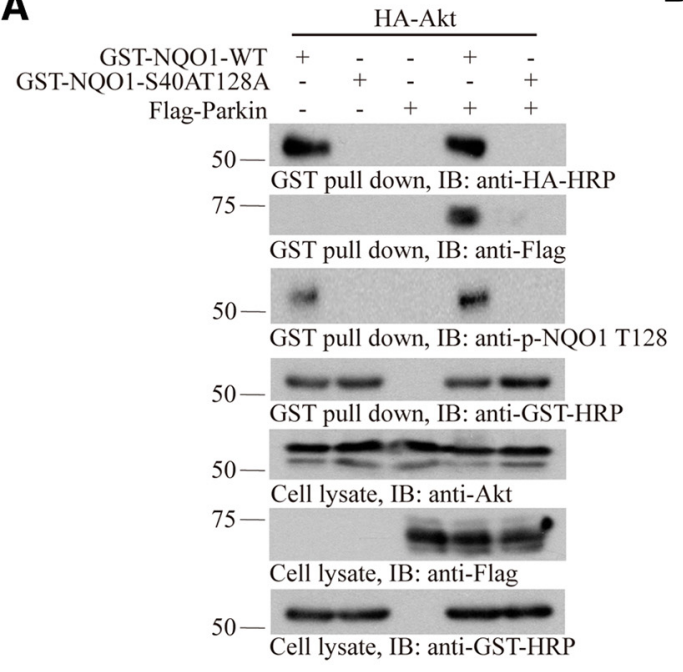

B

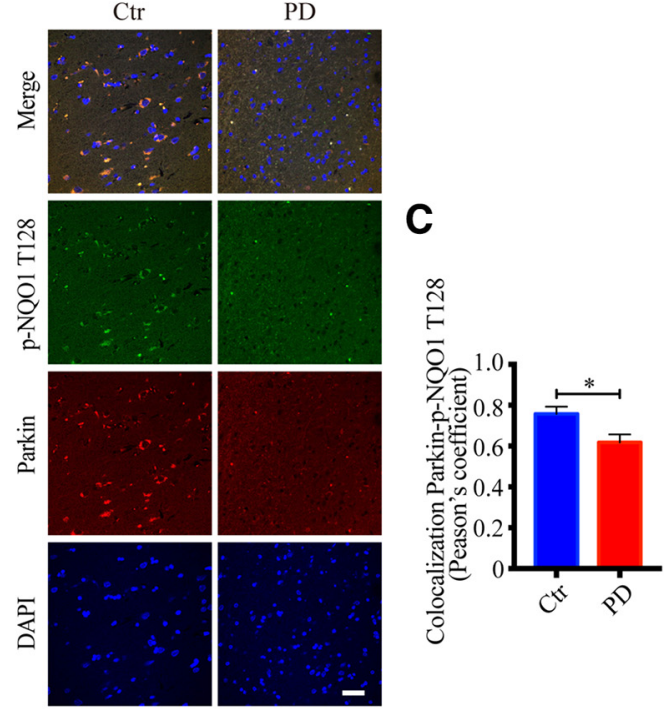

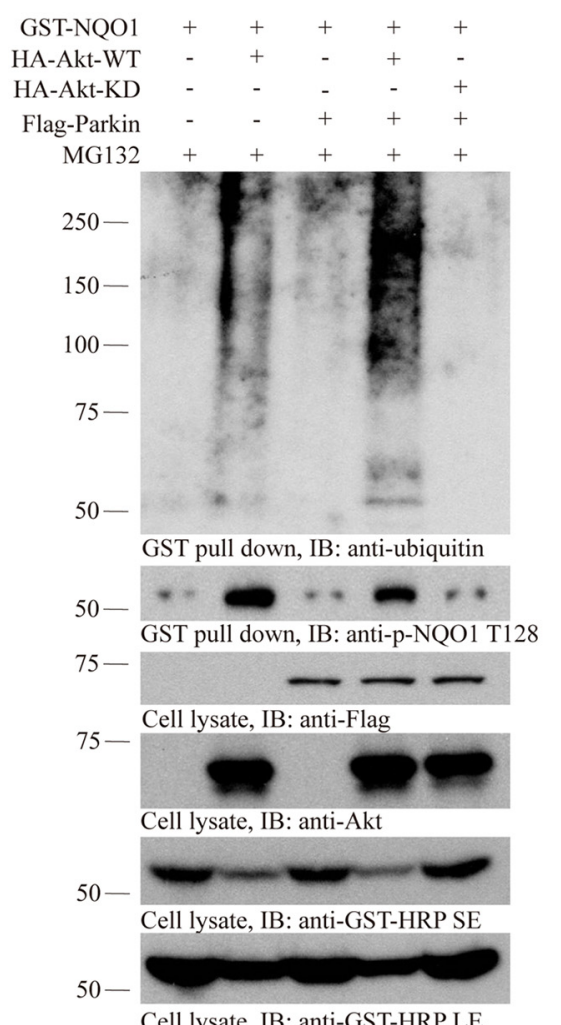

Cell lysate, IB: anti-GST-HRP LE

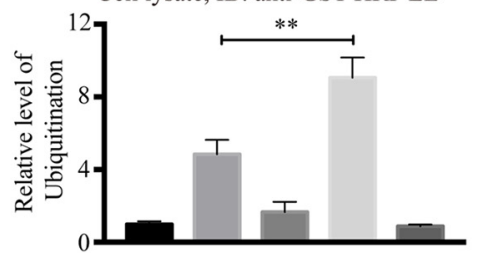

E

SH-SY5Y

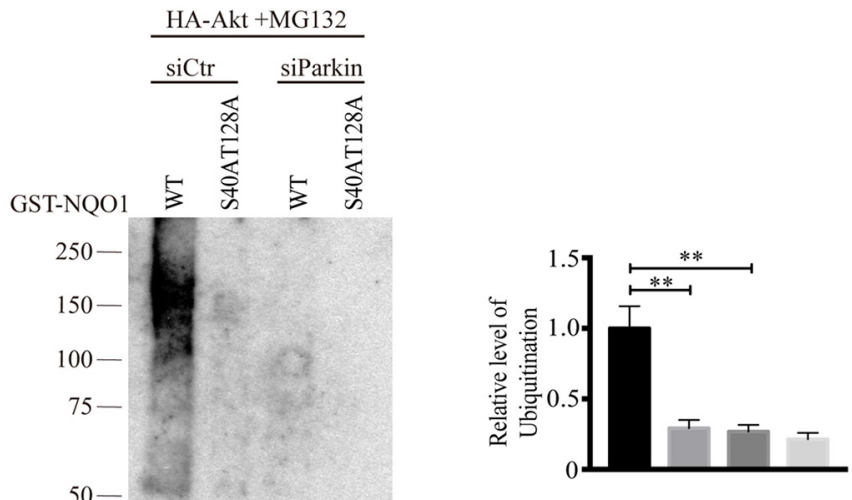

$50-$

GST pull down, IB: anti-ubiquitin
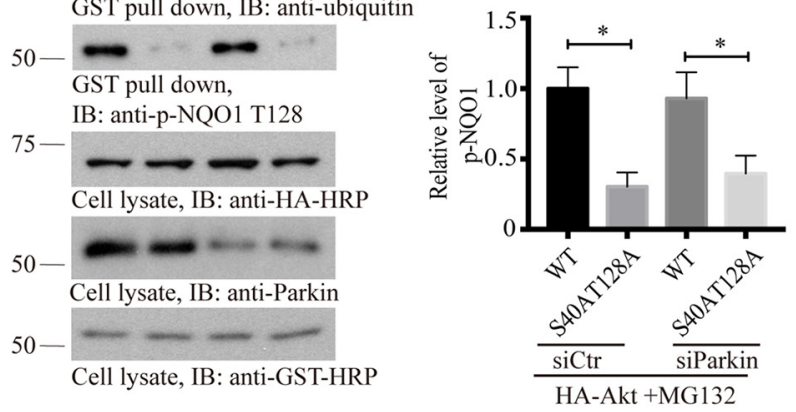

Figure 4. Parkin acts as an E3 ubiquitin ligase for NQ01. A, Parkin associates with NQ01 in a phosphorylation-dependent manner. GST pulldown shows the interaction of Parkin with WT NQ01, but not with the unphosphorylatable S40A/T128A mutant. NQ01 interacted with Parkin regardless of Akt can be found in Figure 4-1A (available at https://doi.org/10.1523/JNEUROSCI.062519.2019.f4-1). B, Immunofluorescent staining of Parkin/p-NQ01 T128 in human PD SN compared with age-matched healthy control. Both Parkin and p-NQ01 were reduced in PD brains. Confocal images show colocalization. Scale bars, $50 \mu \mathrm{m}$. C, Quantification of colocalization in $\boldsymbol{B}$ ( $n=5$ cases per group, $p=0.258$, unpaired $t$ test, two-tailed). $\boldsymbol{D}$, In vitro ubiquitination assay demonstrating that NQ01 ubiquitination was enhanced in the presence of Parkin. NQ01 failed to be ubiquitinated in the absence of Akt phosphorylation. $\boldsymbol{E}$, Knockdown of Parkin abolishes NQ01 ubiquitination. SH-SY5Y cells were pretreated with control or Parkin siRNA for $12 \mathrm{~h}$, followed by transfection with HA-Akt for $36 \mathrm{~h}$ and treatment of MG132 (20 $\mu \mathrm{m}, 3 \mathrm{~h}$ ) twice; then cell lysates were prepared and analyzed by immunoblotting. Right, Quantification of relative ubiquitination and p-NQ01 T128 levels $(n=3)$. For the demonstration that NQ01 ubiquitination is independent of PINK1 involved, see Figure 4-1B (available at https://doi.org/10.1523/JNEUROSCI.0625-19.2019.f4-1). Data are mean \pm SEM. One-way ANOVA with Dunnett's multiple-comparisons test: ${ }^{*} p<0.05$; ${ }^{* *} p<0.01$. Western blot data are representative of three independent experiments. 


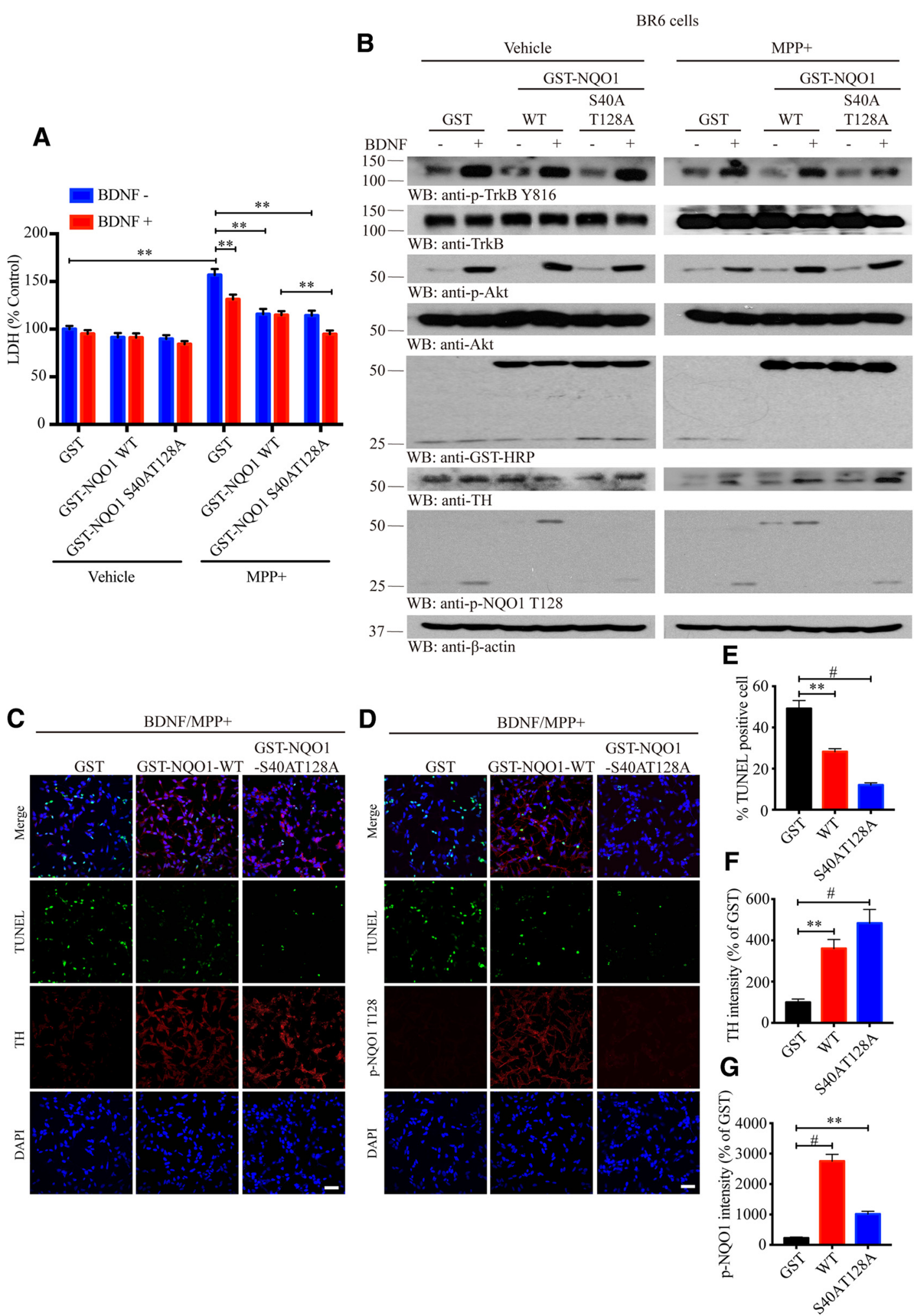

Figure 5. Unphosphorylatable NQ01 mutant possesses stronger cytoprotective effects. $A$, The phospho-dead NQ01 mutant exerts stronger cytoprotective activity. LDH assay showing that NQ01 mutant exhibits stronger cytoprotection after MPP ${ }^{+}$treatment. BR6 cells were transfected with GST-NQ01 for 24 h, followed by BDNF (100 ng/ml, $\left.30 \mathrm{~min}\right)$ twice and then MPP ${ }^{+}(500 \mu \mathrm{M})$ treatment overnight before cell harvesting. Data are mean \pm SEM $(n=3)$. $\boldsymbol{B}$, Western blot analysis showing NQ01 mutant resisted the phosphorylation and degradation by Akt. Blots shown are representative of three independent experiments. The investigation of in vitro antioxidative enzymatic activities of WT and phospho-dead NQ01 can be found in Figure 5-1A (available at https://doi.org/10.1523/JNEUROSCI.0625-19.2019.f5-1). C, D, Immunofluorescent staining of TH/TUNEL and p-NQ01 T128/TUNEL in BR6 cells transfected with GST-tagged WT and mutated NQ01, and then treated with BDNF/MPP ${ }^{+}$. Scale bars, $50 \mu \mathrm{m}$. Quantification of TUNEL-positive cells $(\boldsymbol{E}$, one-way ANOVA, $p=0.038$ and $p<0.0001$, respectively), TH intensity $(\boldsymbol{F}$, one-way ANOVA, $p=$ 0.0051 and $p=0.0002$, respectively), and p-NQ01 T128 intensity (G, one-way ANOVA, $p<0.0001$ and $p=0.0047$, respectively) are shown. Data are mean \pm SEM ( $n=5$ per group). For oxidative stress, evaluation demonstrated that mutant NQ01 possessed the strongest antioxidative activities (see Fig. 5-1B-D, available at https://doi.org/10.1523/JNEUROSCI.0625-19.2019.f5-1). 0ne-way ANOVA or two-way ANOVA with Tukey's multiple-comparisons test: ${ }^{* *} p<0.01 ; " * 0.001$. 
A
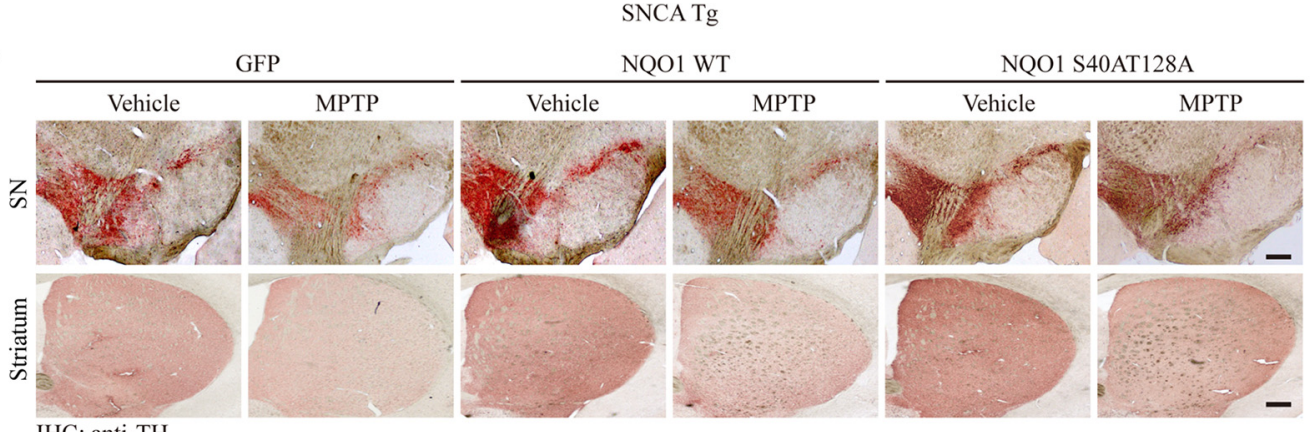

B

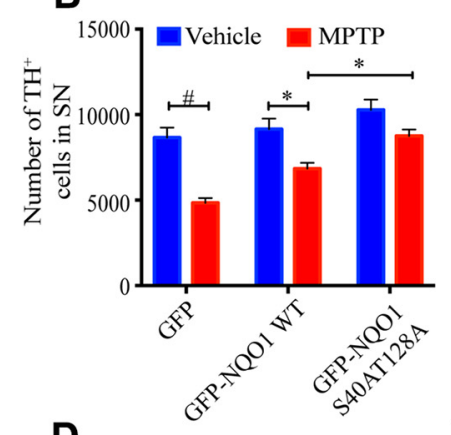

C

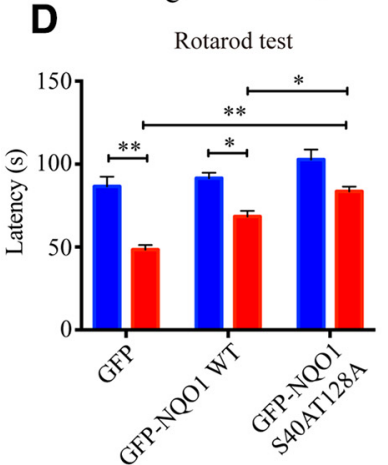

G

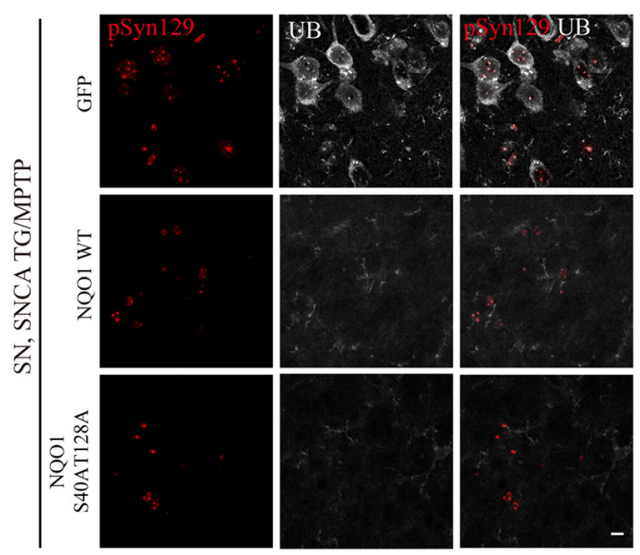

E
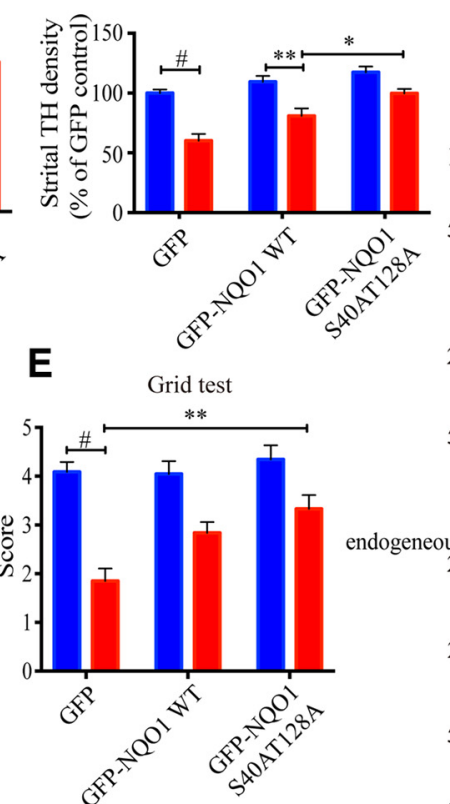

endogeneous-

$\mathbf{F}$

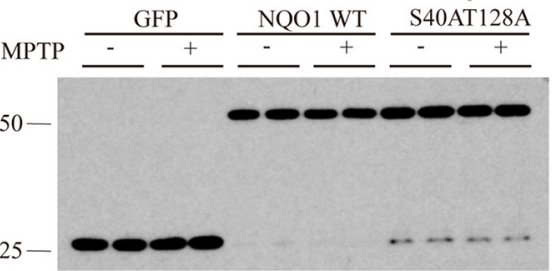

WB: anti-GFP

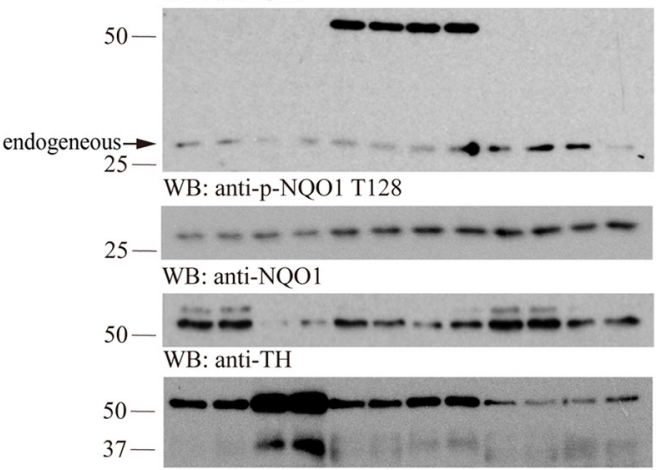

37

WB: anti-AEP

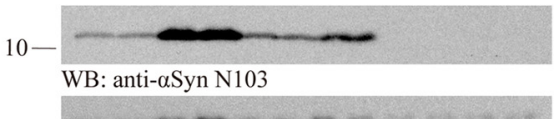

15 - WB: anti-p- $\alpha$ Syn S129

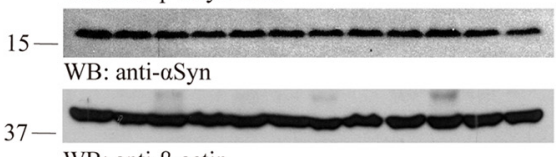

WB: anti- $\beta$-actin

Figure 6. Unphosphorylatable NQ01 mutant protects dopaminergic neurons and rescues motor dysfunction. $A$, Unphosphorylatable NQ01 mutant displays stronger neuroprotective activity than WT NQ01. TH immunostaining of the $S N$ and striatum of the above mice. Scale bars, $200 \mu \mathrm{m}$. Unbiased stereological cell counts in the $S N(B, n=5$ per group, two-way ANOVA, $p<0.0001, p=$ 0.0132 , and $p=0.0403$, respectively) and the total density of striatal dopaminergic terminals $(\boldsymbol{C}, n=5$ per group, two-way ANOVA, $p<0.0001, p=0.0011$, and $p=0.0305$, respectively). Data are mean \pm SEM. Rotarod tests ( $\boldsymbol{D}$, two-way ANOVA, $p=0.007, p=0.0408, p=0.0013$, and $p=0.0485$, respectively) and grid tests $(\boldsymbol{E}$, two-way ANOVA, $p<0.0001$ and $p=0.0033$, respectively) were conducted by a blinded observer 2 months after the virus injection followed by the MPTP treatment. Data are mean \pm SEM ( $n=6-9$ per group). The validation of viral infection efficiency and the evaluation of oxidative stress can be found in Figure 6-1A-C (available at https://doi.org/10.1523/JNEUROSCI.0625-19.2019.f6-1). F, S40A/T128A mutation enhances NQ01's stability and protects dopaminergic neurons. SN lysates were probed with various indicated antibodies. Blot data are representative of three independent experiments. For the detection of AEP activity and the levels of AEP and its product $\alpha$-syn N103 in SN lysates involved, see Figure 6-1D, E (available at https://doi.org/10.1523/JNEUROSCI.0625-19.2019.f6-1). G, Unphosphorylatable NQ01 mutant significantly reduces Lewy body-like pathology in the SN compared with GFP control group after MPTP treatment. Immunofluorescent costaining signals of anti- $\alpha$-synuclein pS129 (red) and anti-ubiquitin (gray) as a Lewy body-like marker were detected in the SN of GFP control mice but completely absent in NQ01 mutant-injected mice. Scale bar, $10 \mu \mathrm{m}$. Two-way ANOVA with Tukey's multiple-comparisons test: ${ }^{*} p<0.05$; ${ }^{* *} p<0.01 ;{ }^{*} p<0.001$. 


\section{Discussion}

In the current study, we show that Akt binds and phosphorylates NQO1 on both S40 and T128 residues, with the latter being the major phosphorylation site. Interestingly, p-NQO1 triggers its ubiquitination and degradation, and NQO1 levels are inversely correlated with p-Akt activities. Notably, Akt, but not canonical PINK1, phosphorylates NQO1 and couples it to the E3 ligase Parkin, which increases $\mathrm{p}-\mathrm{NQO} 1$ polyubiquitination and proteasomal degradation. Remarkably, unphosphorylatable NQO1, due to inhibition of the upstream Akt kinase or S40A/T128A mutation, is much more stable compared with phosphorylated NQO1 WT. Nonetheless, both of them exhibit similar antioxidative enzymatic activities, indicating that phosphorylation does not regulate NQO1 enzymatic activity. Noticeably, we found that both p-NQO1 T128 and Parkin are reduced in human PD brains compared with healthy controls. Moreover, p-Akt levels are also attenuated in synucleinopathies, including DLB and PD patients, so are the upstream neurotrophic BDNF/TrkB signal pathways. Consequently, unphosphorylatable S40A/T128A mutant displays much stronger neuroprotective effect than NQO1 WT in protecting dopaminergic cell survival because the phospho-dead NQO1 mutant is much more stable than its WT counterpart, significantly suppressing ROS and decreasing MPTP-induced neurotoxicity. Hence, blockade of Akt-mediated NQO1 T128 phosphorylation may elongate the half-life of NQO1, augmenting the antioxidative stress in dopaminergic neurons and protecting them from degeneration in PD pathogenesis.

Throughout evolution, cells have developed an efficient protective system involving detoxification and antioxidants. Nrf2, a redox-sensitive transcription factor, is involved in the regulation of the antioxidant response element-mediated expression of Phase II detoxifying antioxidant enzymes, including NQO1 (Favreau and Pickett, 1995). Many antioxidant genes are controlled by Nrf2; however, the gene encoding NQO1 is of particular interest because of its role in the metabolites of DA oxidation-induced oxidative stress (van Muiswinkel et al., 2004). Alterations in the cellular expression of NQO1 in SN have been described as one of the biochemical characteristics of PD (Ross et al., 2000; Beal, 2003). NQO1 levels are increased in the early and intermediate stages of PD. Intriguingly, it is absent at the end stage of the disease, when dopaminergic neurons and extracellular neuromelanin deposits are almost completely disappeared (van Muiswinkel et al., 2004). We also found that $\mathrm{p}-\mathrm{NQO} 1$, Parkin, and p-Akt are prominently reduced in $\mathrm{PD}$ brain compared with control brains (Figs. $1 F$, $2 D, E$, and $4 B, C)$. Moreover, in neurotoxin MPTP models, both p-Akt and NQO1 and its p-NQO1 T128 signals were strongly suppressed in MPTP-treated WT and human $\alpha$-SNCA transgenic mice (Fig. 2-1, available at https://doi. org/10.1523/JNEUROSCI.0625-19.2019.f2-1). Therefore, both NQO1 and its p-T128 levels oscillate in an age-dependent manner in $\alpha$-SNCA mice, and greatly decreased in both PD mouse models and patients.

PTEN-induced putative kinase 1 (PINK1) was initially identified as a PTEN-inducible transcript and contains a serine/threonine kinase domain (Unoki and Nakamura, 2001). Mutations in PINK1 (PTEN-induced putative kinase 1), a familial PDrelated gene, cause an autosomal recessive and early onset $\mathrm{PD}$ (Valente et al., 2001; Bonifati et al., 2005). In addition to regulation of NQO1, Nrf2, an antioxidant transcription factor, mediates PINK1 expression under oxidative stress conditions. Damaged mitochondria arising from stress conditions induced NRF2-dependent transcription of the PINK1 gene through the production of ROS (Murata et al., 2015; Xiao et al., 2017). Mitochondrial function, dynamics, and quality control are vital for the maintenance of neuronal integrity. Indeed, there is mounting evidence that mitochondrial dysfunction plays a central role in several neurodegenerative diseases. Studies on the functions of Parkin and PINK1, which are associated with autosomal recessive Parkinsonism, provide compelling evidence that these proteins can functionally interact to maintain mitochondrial integrity and to promote clearance of damaged and dysfunctional mitochondria (Pilsl and Winklhofer, 2012). The PINK1 gene encodes a 581-amino acid protein that harbors an N-terminal mitochondrial targeting sequence followed by a putative transmembrane region and a serine/threonine kinase domain (Valente et al., 2004). When mitochondria are damaged and lose membrane potential, PINK1 accumulates on the outer membrane of depolarized mitochondria. Accumulated PINK1 recruits Parkin, a PD-linked E3 ubiquitin ligase, and Parkin can mediate the autophagic elimination of depolarized mitochondria (Narendra et al., 2008; Geisler et al., 2010). Biochemical and genetic studies reveal that PINK1 and Parkin normally work together in the same pathway to govern mitochondrial quality control, bolstering previous evidence that mitochondrial damage is involved in $\mathrm{PD}$ (Pickrell and Youle, 2015). Like Akt, PINK1 is an essential prosurvival factor that is induced in response to oxidative stress. PINK1 mutation, knockdown, or KO decreases mitochondrial respiration, mitochondria membrane potential, and ATP generation, and increased ROS, which result in increased susceptibility to apoptosis in various types of cells, including neurons, skeletal muscles, and fibroblasts (Abramov et al., 2011). PINK1 knockdown (KD) or expression of KD PINK1 mutants in PC12 and SH-SY5Y cells decreases ATP generation and oxygen consumption (Beilina et al., 2005; Sim et al., 2006; Liu et al., 2009) and increases ROS production (Gandhi et al., 2009). PINK1 protects cells against various stresses through activation of Akt via mammalian target of rapamycin complex 2 (mTORC2) (Murata et al., 2011; Wu et al., 2013), regulation of Bcl-xL (Arena et al., 2013), and elimination of damaged mitochondria (Matsuda et al., 2010; Narendra et al., 2010). Accordingly, we monitored this pathway in the SN samples and found that Nrf2 and PINK1 expression levels were reduced in an age-dependent manner, partially in alignment with NQO1 levels in $\alpha$-SNCA transgenic mice (Fig. $1 F)$. Conceivably, NRF2 pathway is involved in the oscillating NQO1 expression in PD pathology. Remarkably, we found that Akt, but not PINK1, phosphorylates NQO1 and couples p-NQO1 to the ubiquitin E3 ligase Parkin (Fig. 4).

PI 3-kinase/Akt signaling usually acts as a major pathway mediating survival signals in neuronal cells. Nonetheless, Akt's dual roles in neurodegenerative diseases have been documented. For instance, Akt promotes ataxin-1-induced neurodegeneration. Akt phosphorylates ataxin-1 and elevates its binding to 14-3-3, which in turn leads to ataxin-1 accumulation and neurodegeneration (Chen et al., 2003). Moreover, Akt phosphorylates SRPK2, a cell-cycle regulated pre-mRNA splicing factor, on T492 and promotes its nuclear translocation leading to cyclin D1 upregulation, cell cycle reentry, and neuronal apoptosis in $\mathrm{AD}$. The 14-3-3 binding of SRPK2, regulated by Akt phosphorylation, inhibits these events (Jang et al., 2009). Therefore, the differential effects of PI3-kinase/Akt signaling upon each pathogenic protein exemplify the diversity of cellular responses in different human neurodegenerative diseases. On one hand, NQO1 is induced and upregulated to counteract oxidative stress in the early and intermediate stages of PD. In the meantime, Akt signaling may also be activated to protect neurons from excessive stress. These two 
separate factors may corroborate to promote dopaminergic neuronal survival. However, as the disease progresses, Akt may bind NQO1 and phosphorylate NQO1 at both S40 and T128 residues, which leads the latter to be degraded via polyubiquitination/proteasomal machinery (Figs. 1, 2). The 14-3-3, an adaptor protein coupling Akt and its phosphorylation substrates, can be ubiquitinated by mammalian MrsA in the neurons (Deng et al., 2018). Presumably, 14-3-3 may involve in the interaction between Akt/ p-NQO1, mediating NQO1 polyubiquitination and degradation. Accordingly, the oxidative stress will increase due to lack of antioxidative enzymes, resulting in neuronal cell death. Concomitantly, Akt signaling is also suppressed by various detrimental cellular environments, including aggregated $\alpha$-synuclein and toxic ROS. Ultimately, Akt signaling is reduced in PD brains, and dopaminergic neurons are lost in SN pars compacta (Malagelada et al., 2008; Timmons et al., 2009). Together, our innovative findings support that NQO1 plays a critical role in $\mathrm{PD}$ pathogenesis in response to oxidative stress. Elongating its protein stability may provide an unprecedented therapeutic strategy in treating PD.

\section{References}

Abramov AY, Gegg M, Grunewald A, Wood NW, Klein C, Schapira AH (2011) Bioenergetic consequences of PINK1 mutations in Parkinson disease. PLoS One 6:e25622.

Arena G, Gelmetti V, Torosantucci L, Vignone D, Lamorte G, De Rosa P, Cilia E, Jonas EA, Valente EM (2013) PINK1 protects against cell death induced by mitochondrial depolarization, by phosphorylating bcl-xL and impairing its pro-apoptotic cleavage. Cell Death Differ 20:920-930.

Beal MF (2003) Mitochondria, oxidative damage, and inflammation in Parkinson's disease. Ann N Y Acad Sci 991:120-131.

Beilina A, Van Der Brug M, Ahmad R, Kesavapany S, Miller DW, Petsko GA, Cookson MR (2005) Mutations in PTEN-induced putative kinase 1 associated with recessive Parkinsonism have differential effects on protein stability. Proc Natl Acad Sci U S A 102:5703-5708.

Bonifati V, Rohé CF, Breedveld GJ, Fabrizio E, De Mari M, Tassorelli C, Tavella A, Marconi R, Nicholl DJ, Chien HF, Fincati E, Abbruzzese G, Marini P, De Gaetano A, Horstink MW, Maat-Kievit JA, Sampaio C, Antonini A, Stocchi F, Montagna P, et al. (2005) Early-onset Parkinsonism associated with PINK1 mutations frequency, genotypes, and phenotypes. Neurology 65:87-95.

Bosco DA, Fowler DM, Zhang Q, Nieva J, Powers ET, Wentworth P Jr, Lerner RA, Kelly JW (2006) Elevated levels of oxidized cholesterol metabolites in Lewy body disease brains accelerate $\alpha$-synuclein fibrilization. Nat Chem Biol 2:249-253.

Chen HK, Fernandez-Funez P, Acevedo SF, Lam YC, Kaytor MD, Fernandez MH, Aitken A, Skoulakis EM, Orr HT, Botas J, Zoghbi HY (2003) Interaction of Akt-phosphorylated ataxin-1 with 14-3-3 mediates neurodegeneration in spinocerebellar ataxia type 1. Cell 113:457-468.

Connell RW, Messerschmidt JW (2005) Hegemonic masculinity: rethinking the concept. Gender Soc 19:829-859.

Dehn DL, Siegel D, Swann E, Moody CJ, Ross D (2003) Biochemical, cytotoxic, and genotoxic effects of ES936, a mechanism-based inhibitor of NAD (P) H: quinone oxidoreductase 1, in cellular systems. Mol Pharmacol 64:714-720

Deng Y, Jiang B, Rankin CL, Toyo-Oka K, Richter ML, Maupin-Furlow JA, Moskovitz J (2018) Methionine sulfoxide reductase A (MsrA) mediates the ubiquitination of 14-3-3 protein isotypes in brain. Free Radic Biol Med 129:600-607.

Drukarch B, van Muiswinkel FL (2000) Drug treatment of Parkinson's disease: time for phase II. Biochem Pharmacol 59:1023-1031.

Drukarch B, van Muiswinkel FL (2001) Neuroprotection for Parkinson's disease: a new approach for a new millennium. Expert Opin Investig Drugs 10:1855-1868.

Duffy S, So A, Murphy TH (1998) Activation of endogenous antioxidant defenses in neuronal cells prevents free radical-mediated damage. J Neurochem 71:69-77.

Favreau LV, Pickett CB (1995) The rat quinone reductase antioxidant response element identification of the nucleotide sequence required for basal and inducible activity and detection of antioxidant response element-binding proteins in hepatoma and non-hepatoma cell lines. J Biol Chem 270:24468-24474.

Gandhi S, Wood-Kaczmar A, Yao Z, Plun-Favreau H, Deas E, Klupsch K, Downward J, Latchman DS, Tabrizi SJ, Wood NW, Duchen MR, Abramov AY (2009) PINK1-associated Parkinson's disease is caused by neuronal vulnerability to calcium-induced cell death. Mol Cell 33: 627-638.

Geisler S, Holmström KM, Skujat D, Fiesel FC, Rothfuss OC, Kahle PJ, Springer W (2010) PINK1/Parkin-mediated mitophagy is dependent on VDAC1 and p62/SQSTM1. Nat Cell Biol 12:119-131.

Graumann R, Paris I, Martinez-Alvarado P, Rumanque P, Perez-Pastene C, Cardenas SP, Marin P, Diaz-Grez F, Caviedes R, Caviedes P, SeguraAguilar J (2002) Oxidation of dopamine to aminochrome as a mechanism for neurodegeneration of dopaminergic systems in Parkinson's disease. possible neuroprotective role of DT-diaphorase. Pol J Pharmacol 54:573-579.

Greene LA, Levy O, Malagelada C (2011) Akt as a victim, villain and potential hero in Parkinson's disease pathophysiology and treatment. Cell Mol Neurobiol 31:969-978.

Han JM, Lee YJ, Lee SY, Kim EM, Moon Y, Kim HW, Hwang O (2007) Protective effect of sulforaphane against dopaminergic cell death. J Pharmacol Exp Ther 321:249-256.

Hwang O (2013) Role of oxidative stress in Parkinson's disease. Exp Neurobiol 22:11-17.

Jang SW, Liu X, Fu H, Rees H, Yepes M, Levey A, Ye K (2009) Interaction of Akt-phosphorylated SRPK2 with 14-3-3 mediates cell cycle and cell death in neurons. J Biol Chem 284:24512-24525.

Jazwa A, Rojo AI, Innamorato NG, Hesse M, Fernández-Ruiz J, Cuadrado A (2011) Pharmacological targeting of the transcription factor Nrf2 at the basal ganglia provides disease modifying therapy for experimental Parkinsonism. Antioxid Redox Signal 14:2347-2360.

Kang SS, Ahn EH, Zhang Z, Liu X, Manfredsson FP, Sandoval IM, Dhakal S, Iuvone PM, Cao X, Ye K (2018) $\alpha$-Synuclein stimulation of monoamine oxidase- $\mathrm{B}$ and legumain protease mediates the pathology of Parkinson's disease. EMBO J 37:e201798878.

Kitada T, Asakawa S, Hattori N, Matsumine H, Yamamura Y, Minoshima S, Yokochi M, Mizuno Y, Shimizu N (1998) Mutations in the parkin gene cause autosomal recessive juvenile Parkinsonism. Nature 392:605-608.

Lang AE, Lozano AM (1998) Medical progress: Parkinson's disease. N Engl J Med 339:1044-1053.

Liu W, Vives-Bauza C, Acín-Peréz- R, Yamamoto A, Tan Y, Li Y, Magrané J, Stavarache MA, Shaffer S, Chang S, Kaplitt MG, Huang XY, Beal MF, Manfredi G, Li C (2009) PINK1 defect causes mitochondrial dysfunction, proteasomal deficit and $\alpha$-synuclein aggregation in cell culture models of Parkinson's disease. PLoS One 4:e4597.

Malagelada C, Jin ZH, Greene LA (2008) RTP801 is induced in Parkinson's disease and mediates neuron death by inhibiting Akt phosphorylation/ activation. J Neurosci 28:14363-14371.

Matsuda N, Sato S, Shiba K, Okatsu K, Saisho K, Gautier CA, Sou YS, Saiki S, Kawajiri S, Sato F, Kimura M, Komatsu M, Hattori N, Tanaka K (2010) PINK1 stabilized by mitochondrial depolarization recruits parkin to damaged mitochondria and activates latent parkin for mitophagy. J Cell Biol 189:211-221.

Murata H, Sakaguchi M, Jin Y, Sakaguchi Y, Futami J, Yamada H, Kataoka K, Huh NH (2011) A new cytosolic pathway from a Parkinson diseaseassociated kinase, BRPK/PINK1 activation of AKT via mTORC2. J Biol Chem 286:7182-7189.

Murata H, Takamatsu H, Liu S, Kataoka K, Huh NH, Sakaguchi M (2015) NRF2 regulates PINK1 expression under oxidative stress conditions. PLoS One 10:e0142438.

Nakabeppu Y, Tsuchimoto D, Yamaguchi H, Sakumi K (2007) Oxidative damage in nucleic acids and Parkinson's disease. J Neurosci Res 85:919_ 934

Narendra DP, Jin SM, Tanaka A, Suen DF, Gautier CA, Shen J, Cookson MR, Youle RJ (2010) PINK1 is selectively stabilized on impaired mitochondria to activate Parkin. PLoS Biol 8:e1000298.

Narendra D, Tanaka A, Suen DF, Youle RJ (2008) Parkin is recruited selectively to impaired mitochondria and promotes their autophagy. J Cell Biol 183:795-803.

Orr CF, Rowe DB, Halliday GM (2002) An inflammatory review of Parkinson's disease. Prog Neurobiol 68:325-340.

Pagala VR, High AA, Wang X, Tan H, Kodali K, Mishra A, Kavdia K, Xu Y, 
Wu Z, Peng J (2015) Quantitative protein analysis by mass spectrometry. Methods Mol Biol 1278:281-305.

Pickrell AM, Youle RJ (2015) The roles of PINK1, parkin, and mitochondrial fidelity in Parkinson's disease. Neuron 85:257-273.

Pilsl A, Winklhofer KF (2012) Parkin, PINK1 and mitochondrial integrity: emerging concepts of mitochondrial dysfunction in Parkinson's disease. Acta Neuropathol 123:173-188.

Ross D, Kepa JK, Winski SL, Beall HD, Anwar A, Siegel D (2000) NAD (P) $\mathrm{H}$ : quinone oxidoreductase 1 (NQO1): chemoprotection, bioactivation, gene regulation and genetic polymorphisms. Chem Biol Interact 129: 77-97.

Schultzberg M, Segura-Aguilar J, Lind C (1988) Distribution of DT diaphorase in the rat brain: biochemical and immunohistochemical studies. Neuroscience 27:763-776.

Shimura H, Hattori N, Kubo S, Mizuno Y, Asakawa S, Minoshima S, Shimizu N, Iwai K, Chiba T, Tanaka K (2000) Familial Parkinson disease gene product, parkin, is a ubiquitin-protein ligase. Nat Genet 25:302-305.

Sim CH, Lio DS, Mok SS, Masters CL, Hill AF, Culvenor JG, Cheng HC (2006) C-terminal truncation and Parkinson's disease-associated mutations down-regulate the protein serine/threonine kinase activity of PTEN-induced kinase-1. Hum Mol Genet 15:3251-3262.

Spencer JP, Jenner P, Daniel SE, Lees AJ, Marsden DC, Halliwell B (1998) Conjugates of catecholamines with cysteine and GSH in Parkinson's disease: possible mechanisms of formation involving reactive oxygen species. J Neurochem 71:2112-2122.

Timmons S, Coakley MF, Moloney AM, O’Neill C (2009) Akt signal transduction dysfunction in Parkinson's disease. Neurosci Lett 467:30-35.

Unoki M, Nakamura Y (2001) Growth-suppressive effects of BPOZ and EGR2, two genes involved in the PTEN signaling pathway. Oncogene 20:4457-4465.

Valente EM, Bentivoglio AR, Dixon PH, Ferraris A, Ialongo T, Frontali M, Albanese A, Wood NW (2001) Localization of a novel locus for autosomal recessive early-onset Parkinsonism, PARK6, on human chromosome 1p35-p36. Am J Hum Genet 68:895-900.

Valente EM, Abou-Sleiman PM, Caputo V, Muqit MM, Harvey K, Gispert S,
Ali Z, Del Turco D, Bentivoglio AR, Healy DG, Albanese A, Nussbaum R, González-Maldonado R, Deller T, Salvi S, Cortelli P, Gilks WP, Latchman DS, Harvey RJ, Dallapiccola B, et al. (2004) Hereditary early-onset Parkinson's disease caused by mutations in PINK1. Science 304:1158-1160.

van Muiswinkel F, de Vos RA, Bol JG, Andringa G, Jansen Steur EN, Ross D, Siegel D, Drukarch B (2004) Expression of NAD (P) H: quinone oxidoreductase in the normal and Parkinsonian substantia nigra. Neurobiol Aging 25:1253-1262.

Wang X, Li Y, Wu Z, Wang H, Tan H, Peng J (2014) JUMP: a tag-based database search tool for peptide identification with high sensitivity and accuracy. Mol Cell Proteomics 13:3663-3673.

Wu Z, Sawada T, Shiba K, Liu S, Kanao T, Takahashi R, Hattori N, Imai Y, Lu B (2013) Tricornered/NDR kinase signaling mediates PINK1-directed mitochondrial quality control and tissue maintenance. Genes Dev 27:157-162.

Xiao L, Xu X, Zhang F, Wang M, Xu Y, Tang D, Wang J, Qin Y, Liu Y, Tang C, He L, Greka A, Zhou Z, Liu F, Dong Z, Sun L (2017) The mitochondriatargeted antioxidant MitoQ ameliorated tubular injury mediated by mitophagy in diabetic kidney disease via Nrf2/PINK1. Redox Biol 11: 297-311.

Zafar KS, Inayat-Hussain SH, Ross D (2007) A comparative study of proteasomal inhibition and apoptosis induced in N27 mesencephalic cells by dopamine and MG132. J Neurochem 102:913-921.

Zeevalk GD, Razmpour R, Bernard LP (2008) Glutathione and Parkinson's disease: is this the elephant in the room? Biomed Pharmacother 62: $236-249$.

Zhang Z, Song M, Liu X, Kang SS, Kwon IS, Duong DM, Seyfried NT, Hu WT, Liu Z, Wang JZ, Cheng L, Sun YE, Yu SP, Levey AI, Ye K (2014) Cleavage of tau by asparagine endopeptidase mediates the neurofibrillary pathology in Alzheimer's disease. Nat Med 20:1254-1262.

Zhang Z, Kang SS, Liu X, Ahn EH, Zhang Z, He L, Iuvone PM, Duong DM, Seyfried NT, Benskey MJ, Manfredsson FP, Jin L, Sun YE, Wang JZ, Ye K (2017) Asparagine endopeptidase cleaves $\alpha$-synuclein and mediates pathologic activities in Parkinson's disease. Nat Struct Mol Biol 24:632642. 